\title{
Theoretical Representations of the Built Environment
}

\section{Contents}

6.1 Generalisations on Urban Space and Society …............................................................ 172

6.2 Two Established Research Traditions_-Positivism and Hermeneutics.................................. 173

6.3 Space Syntax's Positivistic Explanatory Models.............................................................. 176

6.3.1 The Theory of Spatial Combinatorics.......................................................................... 176

6.3.2 The Theory of the Natural Movement Economic Process ................................................ 178

6.3.3 The Theory of the Natural Urban Transformation Process................................................ 179

6.3.4 How Space Syntax Allows Theory Building in Line with the Positivism

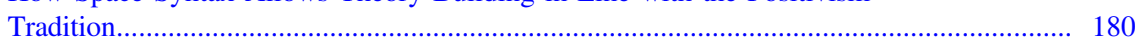

6.4 Space Syntax's Hermeneutic Explanatory Models ............................................................ 181

6.5 Conjunctions and Disjunctions of a Phenomenon ................................................................... 184

6.6 Research Connected to Space Syntax in the Positivism and Hermeneutic

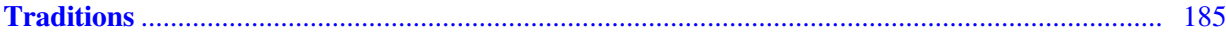

6.6.1 Three Spatial Parameters for Urban Centrality .................................................................. 186

6.6.2 Economic and Cultural Aspects Related to Urban Centrality ............................................ 187

6.6.3 Dealing with Understandings: Context-Dependent Space Syntax Research in Line with the Hermeneutic Tradition .............................................................................. 190

6.7 Context-Dependence: How Space Syntax Theories Can Be Distorted .................................... 198

6.8 A Significant Piece Towards a Comprehensive Theory on the Built

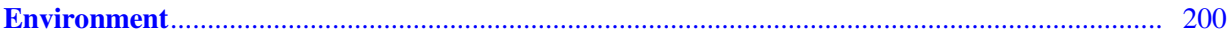

6.9 Epilogue: A Thought Experiment for the Sustainable City Debate ...................................... 201

6.9.1 Describing Compactness with Space Syntax .................................................................. 202

6.9.2 The Street-Building Interface and Its Impact on Street Life and Safety ............................ 204

6.9.3 Spatial Structure, Configuration, and Sustainability .................................................. 207

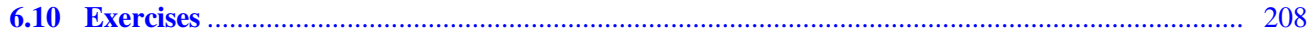

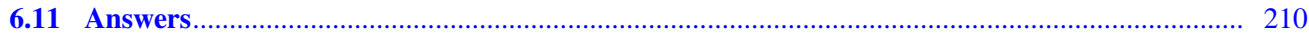

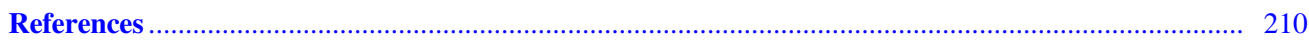

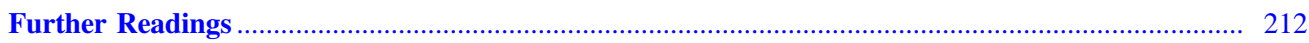

\section{Abstract}

In this chapter, we show what and how space syntax has contributed to theories and general knowledge of the built environment. First, we provide an introduction to two established research traditions, positivism and hermeneutics. The aim is to demonstrate through modal logic what the possibilities and limitations are for gaining general understandings and making theoretical explanations from space syntax research. Modal logic uses expressions to test the explanatory power of statements. Second, we show what space syntax adds to the debate about spatial integration and spatial segregation as seen in relation to market and social rationality. We will focus on the spatial aspects and discuss these in relation to declining versus vital neighbourhoods, crime, anti-social behaviour, cultures, political ideologies, gender, and 
the use of space. Third, we give some reflections on what space syntax has contributed in regards to a comprehensive architecture theory. Finally, at the end, we add as an epilogue a thought experiment on how space syntax theories can be applied within the compact city debate. Exercises are provided at the end of this chapter.

\section{Keywords}

Positivism • Hermeneutics • Explanations $\bullet$ Understandings $\bullet$ Theory building $\bullet$ Generalisation $\bullet$ Urban sustainability $\bullet$

Positivism $\bullet$ Hermeneutics $・$ Modal logic $・$ Explanation $・$ Understanding $\bullet$ Urban theory

\section{Key Concepts}

Positivism • Hermeneutics $・$ Modal logic $・$ Explanation $・$ Understanding $・$ Urban theory

\section{Learning Objectives}

After studying this chapter you will

- distinguish between understandings and explanations, and distinguish which of them belong under the hermeneutic and positivistic research tradition;

- discuss how the three different theories created by space syntax research are able to predict the impacts of spatial interventions;

- test to what extent your research is able to provide explanations or understandings regarding some phenomenon; and

- explain models with the use of necessary and sufficient conditions based on research on built environments with regards to spatial segregation \& integration, various types of urban centralities, space \& society, and spatial cultures.

\subsection{Generalisations on Urban Space and Society}

Space syntax and its variants are constantly developing, and developments in computer science allow for improvements in the formal applications that the space syntax tools rely on. In particular, the increasing number of context-dependent case studies calls for a refined application and adequate interpretation of space syntax methods. Continuous research and its lasting results depend on methodological reliability and on a systematic accounting of the conditions under which the claims might turn out to be true or false. This is what theory development on built environments is dependent on, and thus how space syntax methods have been improved and developed over the last three decades.

While the social science disciplines have been well established, for urban sciences in general theory development and understandings of the role of the spatial component are still in an early phase. In urban sciences, theory development only started in the twentieth century, and much still needs to be done in terms of refining the definition of spatial components, performing empirical tests, and making generalisations and theories applicable in urban design and planning. Therefore, we introduce in Sect. 6.2 two established research traditions relevant to the urbanism disciplines. In Sect. 6.3, we show which parts of the space syntax research belong under the positivistic research tradition. The various theories and explanations developed with space syntax research are discussed here. In Sect. 6.4, we show which parts of space syntax research belong under the hermeneutic research tradition. Here the various understandings of human intentions, of causes, and of various cultures are discussed. In Sect. 6.5, we provide some complex explanatory models for both the hermeneutic and positivistic traditions.

The use of space syntax has contributed to an understanding of the spatial structure of the city as an object (Hillier 2001) shaped by a society, as well as to knowledge on how the physical layout of the built environment can generate or affect certain socio-economic processes in a society. To some extent, space syntax is able to predict some types of economic processes as an effect of urban interventions. Likewise, space syntax provides understandings of the spatial possibilities for certain social activities such as crime, social segregation, and anti-social behaviour. It is all about how spatial integration and segregation condition sial integration and segregation. These issues will be discussed in Sects. 6.6 and 6.7. In Sect. 6.8, we reflect on what space syntax's contribution is towards a comprehensive theory on the built environment. Finally, in an epilogue section, we reflect on how space syntax can be used in the compact city debate. 


\subsection{Two Established Research Traditions-Positivism and Hermeneutics}

Theory building on the built environment is still at a beginning phase in comparison with the theories of the sciences. The development of scientific theories started around 4,000 years ago, whereas descriptive research on the built environments started only in the 1920s, with the work of the urban sociologists at the Chicago school. Therefore, methods from elementary theories of science are useful for developing theories and for explaining how the built environment works.

There exist two established, but different, research traditions_-positivism and hermeneutics. These two research traditions influence how we can gain explanations and understandings about the relation between the built environment and society. Space syntax research is interdisciplinary, and when dealing with research on urban artefacts, urban form, and urban space, these aspects lie at the overlap between the human, social, and natural sciences. We are dealing with research on the relationship between society, its members' cognitive aspects, and the physical 'framework' within which all of these activities take place. Therefore, general understandings and theory building on the built environment require a hermeneutic (dealing with intentional explanations) as well as a positivistic (dealing with causal explanations) approach.

The positivistic research tradition is rooted in the natural sciences. The focus is mostly on objects free from intentions and the meanings attached to them. Through experiments, observations, systematic testing, and technical interventions, operational theories are developed. These theories give insights into nature and technical applications. Most of their explanatory models, for example, the hypothetico-deductive method, are related to cause and effect (Føllesdal et al. 1996, p. 94). The hypothetico-deductive method allows for the formulation of a falsifiable hypothesis that can be tested on observable data where the outcome is not yet known. In other words, the hypothetico-deductive method consists of formulating a hypothesis and deducting the conclusion based on the premises. Consider the example below:

Hypothesis: If $A$, then $B$.

Premise 1: Not $B$.

Conclusion 1: not $A$.

Premise 2: $B$

Conclusion 2: $A$

Premise 1 and 2 can be verified through observations, and from there the conclusion can be derived. Research belonging under the positivism tradition deals with objects that are free from human intentions and meanings. Research of this kind has a high degree of predictability and is able to explain the phenomena around us in the field of the natural sciences. Therefore, the model above can easily be applied in Hillier's theories on spatial combinatorics (see Sect. 6.3).

Hypothesis: If an object is placed in a middle of a square, then the topological depth is increased.

Premise 1: The topological depth is not increased.

Conclusion 1: The object is not placed in the middle of the square.

Premise 2: The topological depth is increased.

Conclusion 2: The object is placed in the middle of the square.

Another example of the hypothetico-deductive method is the example below:

Hypothesis: All $A$ are $B$.

Premise: $c$ is $A$.

Conclusion: $c$ is $B$.

Again, we can apply this example to Hillier's theory on spatial combinatorics for the case of the Victory Column in Berlin (Fig. 6.1).

Fig. 6.1 The Victory Column in Berlin: a space syntax analysis of the street network without the column (a) and with the column (b)

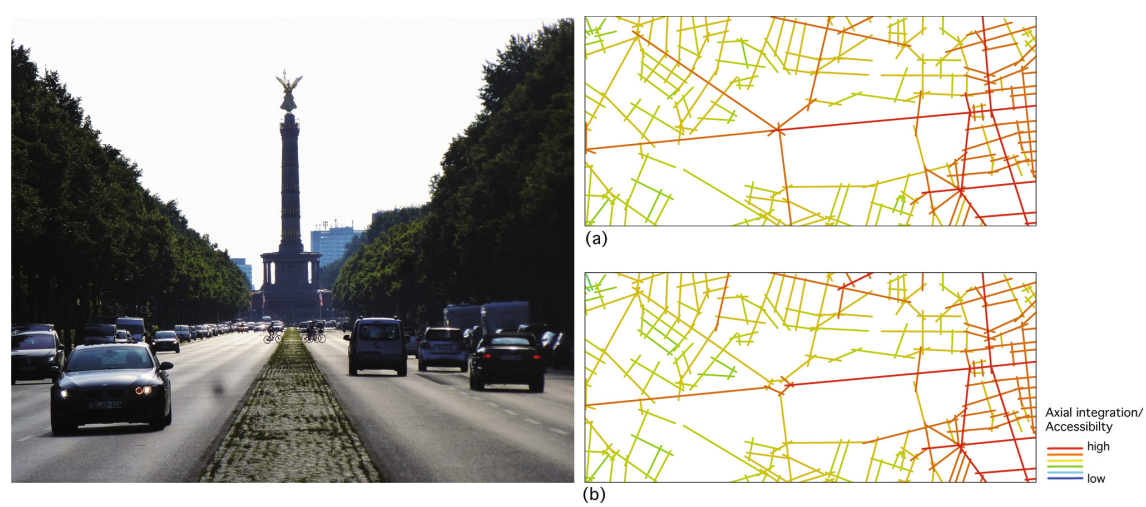


Hypothesis: All objects placed in an open urban space increase the topological depth.

Premise: The Victory Column in Berlin is an object placed in the middle of the street Bundestraße 2.

Conclusion: The Victory Column in Berlin increases the topological depth of Bundestraße 2.

The success of the natural sciences contributed to several attempts to imitate its positivistic approaches in the human and social sciences, but without success. In the 1850s, German researcher Johann Gustav Droysen (1808-1884) noted that the aim of the natural sciences is to explain a phenomenon, whereas in the human and social sciences the aim is to understand a phenomenon. Here, the aim is to understand the motives and intentions behind the actions of the persons involved, to understand the action itself, and to investigate the results of these actions (Føllesdal et al. 1996, p. 95). Most research in the field of urban morphology and place phenomenology has focused on gaining an understanding of the built form as a product and an understanding of the meanings or memories attached to it.

The concept of 'hermeneutics' was introduced in the early seventeenth century, but its development as a research tradition took place in the nineteenth century through the work of German scholars Friedrich Schleiermacher (1768-1834) and Wilhelm Dilthey (1833-1911). In hermeneutics, the hypothetico-deductive method is applied to writings or objects that have meanings attached to them. The aim is to understand or to derive the original meanings and human intentions behind the material. Thus, hermeneutics is an interpretational approach. From the 1960s onwards, a new hermeneutic direction was developed by Hans-Georg Gadamer and Jürgen Habermas. Herein, Gadamer's concept 'fusion of horizons' (German: Horizontverschmelzung) was developed. It is defined as the sum of the impressions and attitudes we have at a certain moment, including those that are conscious, as well as those that are unconscious and thus not a target of our focus (Føllesdal et al. 1996, p. 101).

The difference between the 'traditional' hermeneutics and the 'new' hermeneutics is that scholars in the new hermeneutics acknowledge that the researcher can never free themself from their own understanding horizon. This is referred to by Gadamer as "the universal challenge in hermeneutics" (Føllesdal et al. 1996, p. 107). In general, the hermeneutic method consists of interpretation cycles, where the researcher has to interpret the available materials in terms of the meanings and intentions of past and present society. The fusion of horizons increases through reflections (Føllesdal et al. 1996, p. 105).

The challenge is to strive for objective knowledge. Popper claims that objective knowledge depends to a high degree on the falsifiability of hypotheses and on the methods that are used (Popper 1963, p. 39). Perception practice operates with two types of information that are relevant for falsification, namely subjective and objective knowledge. Subjective knowledge depends on cultural and personal values and how a built environment is perceived. The objective information in this context concerns geometrical and topological descriptions of the built environment's spaces and available socio-economic data. Quantitative socio-economic data can be correlated and tested against the spatial parameters resulting from the space syntax method.

In urban research, interpretation of the meaning and memory of urban artefacts and urban patterns and the understanding of society over time both belong under the hermeneutic research tradition. This is all connected to the researchers' own attitudes. We can also identify hermeneutic approaches within space syntax research, especially those approaches linked to research topics concerning socio-environmental disorder, history, archaeology, anthropology, and cultural tradition with an impact on urban space as well as religious activities in relation to space (Van Nes 2017; Aleksandrowicz et al. 2017, 2018). Within these fields, predictability is not embedded and therefore no theories or explanatory models with predictable power have been outlined.

Research on the built environment deals with the physical aspects of the environment, as well as with the meanings, intentions, and actions of human beings within the environment. Therefore, both research traditions are appropriate in the space syntax field. The reasons are as follows. First, the space syntax method separates definitions of spatial elements from human intentions and the memories attached to them. Second, the calculation methods deal solely with spatial objects. Therefore, space syntax is able to generate positivistic explanatory models for space and spatial relationships. The correlation of space syntax results with socio-economic data requires an understanding of human intentions and actions, and therefore, involves a hermeneutic approach.

Both research traditions need different explanatory models and are applicable for space syntax research. Interestingly enough, human intentions related to time and economic efficiency make it possible to derive causal explanatory models in line with the positivistic tradition. Following this, we explore the explanatory power of both research traditions using the philosopher Georg Henrik von Wright's modal logics with examples from space syntax research. Modal logics are a type of formal logic that extends the classic logic to include modality. Modality concerns a system's lingual options for expressing the general intentions of a society.

Von Wright has clarified how the notions of causal and intentional explanations depend on the positivist and the hermeneutic traditions. Both of these epistemological traditions impose different structures and conditions on their explanatory models. Epistemology is the study of the nature of knowledge justification and the rationality of belief. Von 
Wright states that the way his modal logic models are built up depends on the types of conditions, and how they can be used to explain or understand a particular phenomenon (von Wright 1971, pp. 1-3). The positivist tradition emphasises the identification of the causes of a certain phenomenon. Therefore, research belonging under the positivistic tradition has a high degree of predictability. Their explanatory models anticipate nothing other than explaining the effects of certain causes (von Wright 1971, p. 3). Most research in the natural sciences belongs to the positivist tradition and uses causal explanatory models.

In contrast, the hermeneutic tradition emphasises explanations that search for an understanding beyond the phenomenon (von Wright 1971, p. 4). Most research in the human and social sciences belongs to the hermeneutic tradition, and therefore, uses intentional explanatory models. Research on the built environment is a combination of both traditions and incorporates aspects from the natural sciences and the social sciences. Therefore, we need to understand and explore the potentials and limitations of explanatory models embedded in both the hermeneutic and positivist traditions.

For his explanatory models, von Wright distinguishes between necessary and sufficient conditions. The use of these kinds of conditions determines whether the explanatory model is applied to a hermeneutic or a positivist form of research (von Wright 1971, p. 38). His terminology refers to causal explanations from a positivist perspective as simply 'explanations' and in a hermeneutic context as 'understandings'. 'Explanations' relate to sufficient conditions accounting for the causes of certain effects. 'Understandings' result from an assessment of necessary conditions reasonably associated with causes. Causes precede their effects. The notion of time is a decisive, though certainly not sufficient, criterion for the distinction between causes and effects (von Wright 1971, p. 41).

The current challenge for theory building in space syntax is to be aware of the relationship between cause and effect and the intentions behind a cause. Figure 6.2 shows where the focus on the explanation is in these two research traditions. Von Wright's modal logics contribute to defining the limitations and possibilities to make general theories and show the situations where we cannot make theories and are limited to understandings.
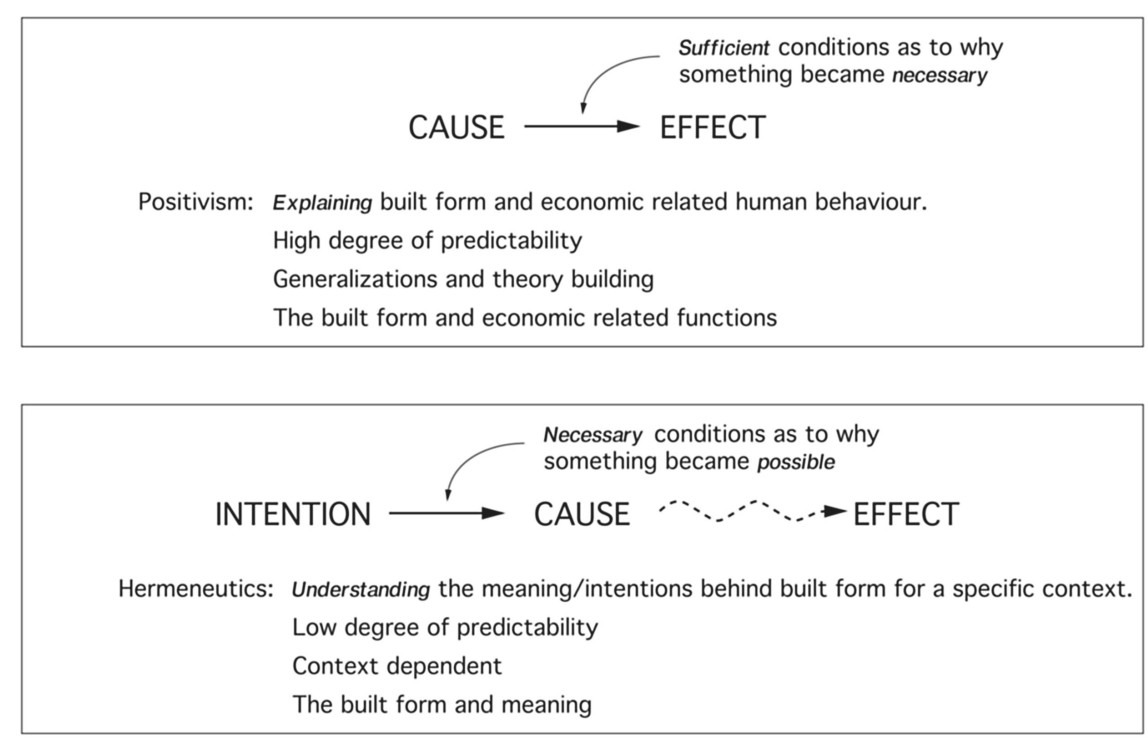

Fig. 6.2 The different focus on the explanations from the positivistic and hermeneutic research traditions

Research into activities in society and how they impact the shaping and forming of the built environment requires a hermeneutic approach. Herein, the purpose is to gain an understanding of how activities in society influence the urban form. Research of this kind is context-dependent, and therefore, it is difficult to develop a generalised theory on a society's influence in producing a particular spatial environment. Conversely, how a built environment's spatial layout affects human behaviour requires both a positivistic and a hermeneutic approach. For research with regards to space and market rationality, i.e. profit maximising, a positivistic approach makes it possible to gain explanations into how a built environment's spatial layout affects activities in society with regards to movement and to the location of economic-related activities. 


\subsection{Space Syntax's Positivistic Explanatory Models}

In this section, we will demonstrate how some existing theories from space syntax fit into the positivistic explanatory models from von Wright when addressing spatial changes as an effect of physical interventions. Until now, most space syntax research has focused on explaining how a city is set up as an object, irrespective of human intentions and meanings. For this purpose, the built environment is perceived as a set of spaces shaping a configurative spatial system. Each physical change in the built environment affects its configurative spatial system. This is, in contrast, to research in the urban morphology and place phenomenology traditions that are closely intertwined with understanding the human intentions behind artefacts. When dealing with human rationality where intentions are unambiguous, it is possible to predict the effect on human behaviour caused by spatial changes. Market rationality and all kinds of rationality dealing with time and cost efficiency have unambiguous intentions (Van Nes 2017), which makes explanations and theory building possible in line with the positivist research tradition.

There exist three descriptive theories on built environments that are in line with the positivistic research tradition:

(1) The theory of spatial combinatorics (Hillier 1996, Chap. 8)

(2) The theory of the natural movement economic process (Hillier et al. 1993, 1998)

(3) The theory of the natural urban transformation process (Ye and Van Nes 2014)

\subsubsection{The Theory of Spatial Combinatorics}

This theory deals with calculating spatial combinatorics and deals only with the spatial aspects of the built environment itself. Therefore, human intentions, meanings, and memories are not considered. Through the calculations from the two-dimensional point depth analyses, some elementary principles on urban centrality can be derived. The way public spaces are blocked or the way main routes are free from physical obstacles influences how city centres emerge or fade away (Hillier 1996, Chap. 8). With regard to spatial combinatorics, Fig. 6.3 illustrates two principles regarding what happens when a physical object is placed in an urban space. Here, the principle of centrality is applied and demonstrates that a centrally placed object increases the mean depth more than an object placed at the edge of the geometric figure. In other words, placing a large building in the middle of a central square contributes to the overall segregation of the neighbourhood more than placing the same building at the square's edge. Furthermore, the principle of extension explains that partitioning a longer urban space, e.g. a long street increases the mean depth value more than partitioning a shorter urban space. This implies that blocking long main routes that connect a city's centre to its edges contributes to segregating a city more than blocking short residential streets.

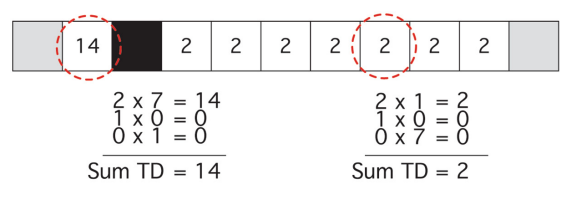

Total depth $(\mathrm{TD})$ gain $=28$

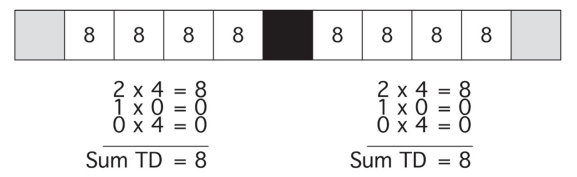

Total depth $(\mathrm{TD})$ gain $=64$

The principle of CENTRALITY - a centrally placed object increases the depth more than one placed at the edge

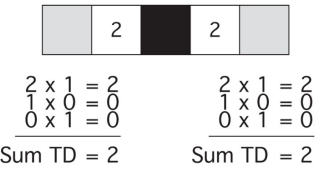

Total depth $(T D)$ gain $=4$

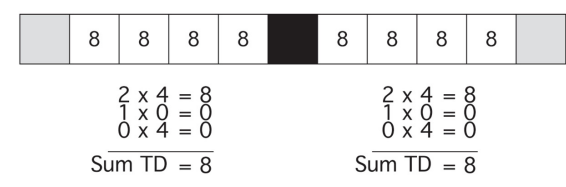

Total depth $($ TD) gain $=64$

The principle of EXTENSION - partitioning a longer line increases the depth more than partitioning a short line

Fig. 6.3 The principle of centrality and extension (redrawn; original: Hillier 1996, Chap. 8) 
These principles are in line with how cities grow naturally. A city consists of a very large number of short streets and a very small number of long streets. The function of the long streets is for wayfinding, so that people can orientate themselves from the edges to the centre. Therefore, these long streets are seldom blocked by artefacts when cities grow.

From this principle, we can derive a spatial logic for compact or non-compact cities. Figure 6.4 depicts two principles for what happens in public space when placing different geometric objects in the built environment. The principle of contiguity explains that continuous blocks increase the mean depth value more than separate blocks. This implies that smaller urban blocks contribute to higher spatial integration than large urban blocks. The principle of compactness states that straight blocks increase the mean depth value more than curved blocks. In other words, long building blocks contribute to segregating a neighbourhood more than compact curved buildings. These simple spatial models are the first steps for explaining how various urban spatial configurations provide various degrees of accessibility. Seemingly, the invisible spatial structure between physical objects is something 'active' and has its own laws shaping limitations or possibilities for movement. These simple spatial models contribute to theory building on urban space by providing descriptive principles on spatial 'drivers' for urban centrality.

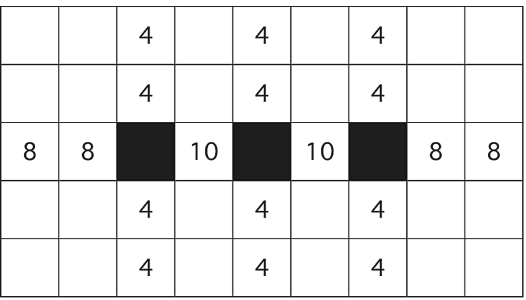

Total depth gain $=100$ for 18 cells

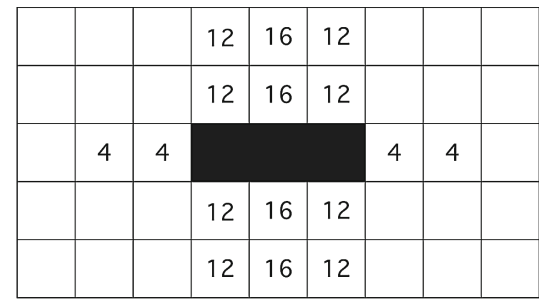

Total depth gain $=176$ for 16 cells

The principle of CONTIGUITY - contiguous blocks increase depth more than separate ones

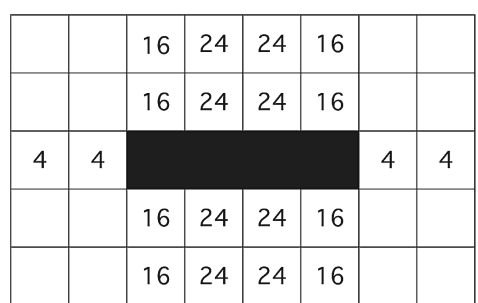

Total depth gain $=336$ for 20 cells

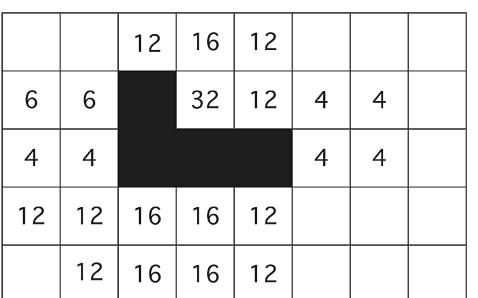

Total depth gain $=256$ for 23 cells

\section{The principle of COMPACTNESS - straight lines} increase depth more than "curved" lines

Fig. 6.4 The principles of contiguity and compactness (redrawn; original: Hillier 1996, Chap. 8)

Figure 6.5 shows some examples of different building types affecting the size of urban blocks. Image (a) shows an example of the high-rise flats from the 1960s in a suburb of Amsterdam. The building type contributes to large urban blocks and to a low degree of permeability for people moving through this suburb. Further west in the same suburb, Bijlmermeer, case (b), the "u"-shaped low-rise urban flats contribute to smaller urban blocks compared to case (a). Case (c) shows an image of a suburb in Moscow close to the international airport. The shape of the flats and the urban blocks has similarities to case (a). Case (d) shows the historic city centre of Brasov consisting of small buildings and small urban blocks. This urban area has a high degree of permeability. 
Fig. 6.5 Some examples of a compact and non-compact urban footprint: (a) high-rise flats in Bijlmermeer in Amsterdam; (b) low-rise flats in Bijlmermeer in Amsterdam; (c) high-rise flats in Moscow; and (d) the historic core of Brasov in Romania
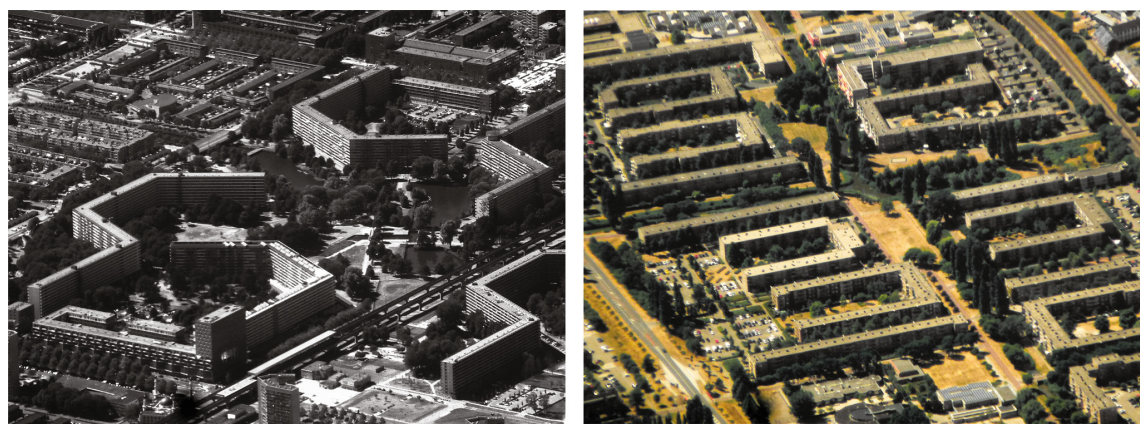

(a)
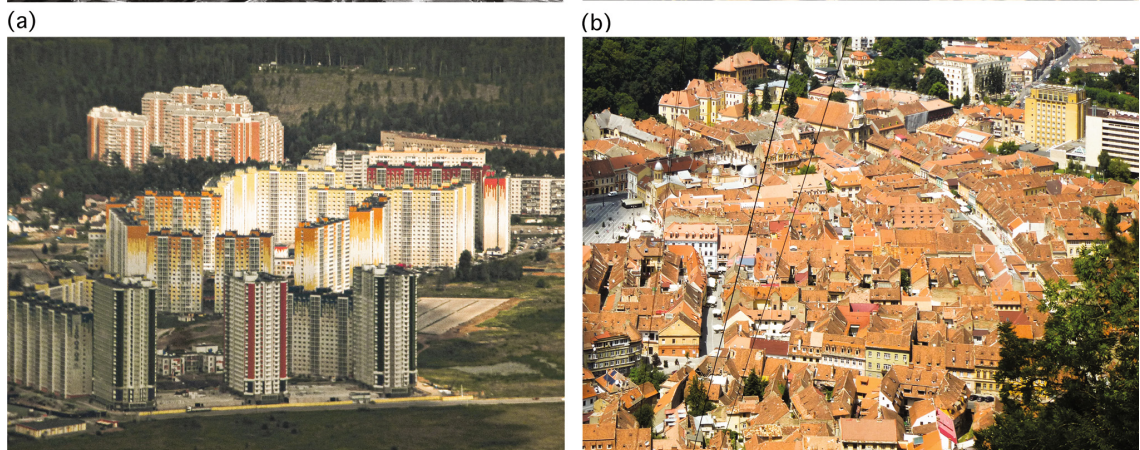

(d)

Hillier formulated four principles for the theory of spatial combinatorics that have a clear relationship between cause and effect. These four spatial principles are connected to the geometry of urban blocks, to where they are placed in space, and in what kind of street they are located. Together the placements of physical objects in urban space influence the degree of inter-accessibility, which again is connected to the degree of centrality, urbanity, or anti-urbanity of an urban system. The four principles are:

(1) Principle of centrality: a centrally placed object increases the topological depth more than one placed at the edge.

(2) Principle of extension: Partitioning a longer line increases topological depth more than partitioning a short line.

(3) Principle of contiguity: Continuous, large blocks increase topological depth more than smaller, separate blocks.

(4) Principle of compactness: Straight lines increase topological depth more than 'curved' lines.

These principles are based on mathematical calculations of spatial relationships. They can be linked to Jane Jacob's presumptions (1961) that large continuous urban blocks contribute to longer walking distances compared to short urban blocks. Whereas Jacobs takes into consideration the size of the urban block, space syntax analysis focuses on how the size and shape of the block affects the degree of inter-accessibility of the spaces between these blocks. Spatial inter-accessibility is linked to the theory of the natural movement economic process.

\subsubsection{The Theory of the Natural Movement Economic Process}

The theory of the natural movement economic process states that the spatial configuration of the street and road network influences the flow of human movement and the location of shops in built environments. The higher the spatial integration of the street network, the higher the flow of human movement, and the more the land along the street network becomes attractive for economic uses. The more people are in the streets, the more shops tend to locate along these streets, and the more shops there are along these streets, the more people are attracted to the area (Hillier et al 1993, 1998). This process changes if the spatial configuration of the street network is changed through physical interventions such as walls (Desyllas 2000) or new road or street links (Van Nes 2002; Van Nes 2021). Figure 6.6 shows the relationship between configuration, attraction (the location of shops), and movement. This theory explains how the built environment functions independently of 
planning processes with regard to the location pattern of economic activities, human movement through the urban network, and the spatial configuration of the built environment. After all, movement and attractors do not influence the street network's spatial configuration, and instead, it is the street network's spatial configuration that affects the flow of movement and the optimal location for economic activities. For this theory there are four kinds of cause-effect relationships:

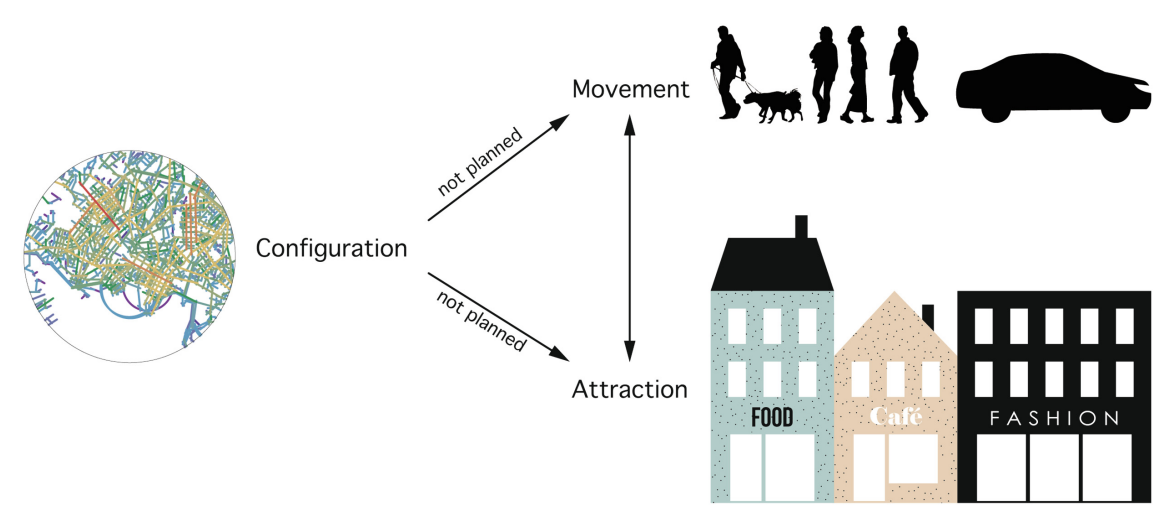

Fig. 6.6 The theory of the natural movement economic process

(1) The spatial configuration of urban space affects the flow of human movement.

(2) The spatial configuration of urban space affects the location pattern of economic activities.

(3) The amount of human movement influences the location pattern of economic activities in the built environment.

(4) The location pattern of economic activities influences the amount of human movement in the built environment.

\subsubsection{The Theory of the Natural Urban Transformation Process}

The theory of the natural movement process (Hillier et al. 1993, 1998) provides the basis for the theory of the natural urban transformation process (Ye and Van Nes 2014). This theory states that the spatial configuration of the street and road network influences the degree of building density and the degree of multi-functionality of land use. The higher the overall spatial integration of the mobility network on various scales, the higher the building densities (both for the Floor Space Index (FSI) and Gross Space Index (GSI)) and the higher the diversity in land use. Seemingly, the spatial configuration of the street network, as the foundation for steering urban transformations, influences the degree of building density and land use diversity. Likewise, the degree of building density influences in the long term the degree of land use diversity. The first assumptions of these correlations were made after 2010 (Hausleitner 2010; Van Nes et al. 2012) and finally clarified in 2014 (Ye and Van Nes 2014). This theory contrasts with current planning practice in America and in several European and Asian countries. Figure 6.7 shows the relationship between configuration, building density (in terms of FSI and GSI), and degree of land use mixture.

In summary, the theory of the natural urban transformation process explains how a built environment functions independent of planning processes with regard to restrictive land use plans, requirements for maximum or minimum FSI or GSI, and the spatial configuration of the street network. The degree of land use mixture depends on both the degree of building density and the spatial configuration of the street network. For this theory, there are three cause-effect relationships: 
Fig. 6.7 The theory of the natural urban transformation process

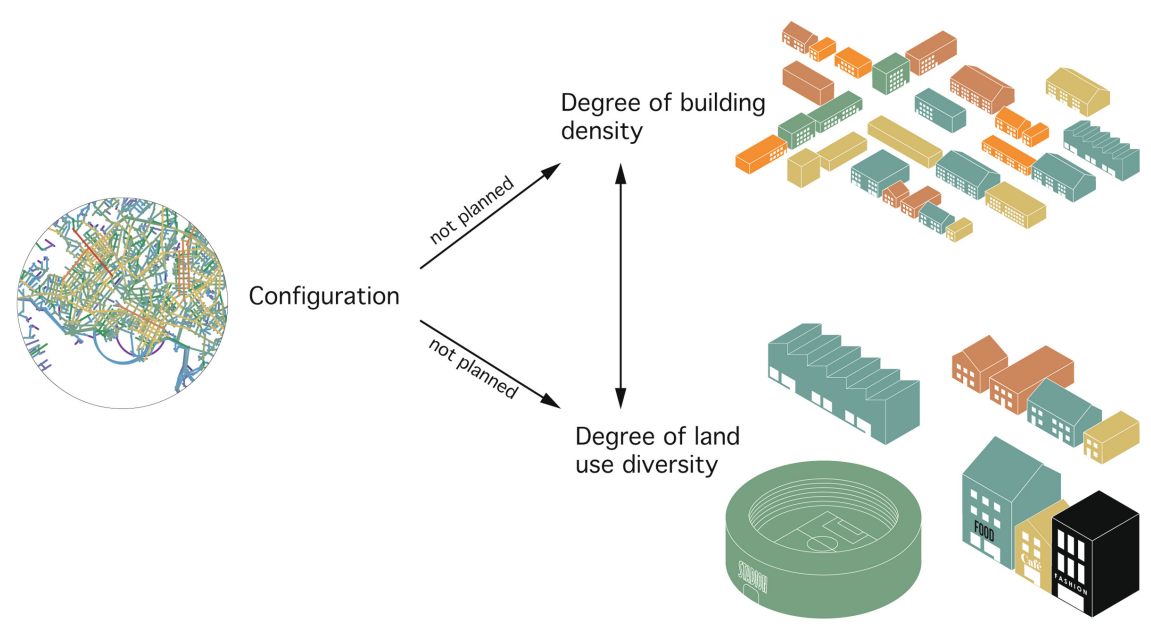

(1) The spatial configuration of the urban space affects the degree of building density.

(2) The spatial configuration of the urban space affects the degree of land use diversity.

(3) The degree of building density influences the degree of land use diversity.

Explaining cause-effect relationships clearly belongs under the positivism tradition. In the next section, we will present the conditions between cause and effect for testing the explanatory power of various statements based on space syntax theories. Here we go back again to elementary theories of science.

\subsubsection{How Space Syntax Allows Theory Building in Line with the Positivism Tradition}

Causal explanatory models seem appropriate for assessing the explanatory power of the theory of spatial combinatorics (Hillier 1996, Chap. 8), the theory of the natural movement economic process (Hillier et al. 1998; Hillier 1999a, b; Van Nes 2002), and the theory of the natural urban transformation process (Ye and Van Nes 2014). The nature of all three theories is that they have cause-effect relationships as their underlying logic.

If we apply the theoretical logic of space syntax to von Wright's causal explanatory model (1971), we see the strength of space syntax theory with regard to generalised theory building. We illustrate this by the application of space syntax to real-life urban examples. The explanation of cause-effect relationships is theory building, but when dealing with intentions behind a cause, one is dealing with understandings, which require context-dependent cases. Space syntax allows both explanations and understandings but depends on the kind of rationality at hand and to what extent we are dealing with only urban space or with urban space and society.

\section{General}

Effect (= consequence): The spatial configuration of an urban street network is changed.

Cause (= reason): A new movement route was built.

\section{Context-dependent}

Effect (= consequence): The integration values of Friedrichstraße in Berlin increased in 1990.

Cause (= reason): The removal of the Berlin Wall changed the spatial configuration of Berlin's street network.

The above examples illustrate the explanatory power of research results dealing only with physical aspects of the built environment. Also, when adding unambiguous human intentions, both general and context-dependent examples work well. 
The intentions of market rationality are clear, namely profit maximising —in the sense of Homo economicus-and time efficiency. Therefore, a high degree of predictability can be derived when the spatial layout of the city affects the efficient location of economic-related activities. In general, the development of scientific theories aims at an increasing degree of generality. Hence, only general examples are presented in terms of von Wright's explanatory models for research related to a market rationality. In the following, we use examples from street network research and discuss, based on von Wright's explanatory model, whether a new movement route is a sufficient or necessary condition for changing the spatial configuration system in a city.

Positivism explanatory model

$p$ is a sufficient condition of $q$.

A new road link is a sufficient condition for a change in the spatial configuration system.

Hermeneutic explanatory model

$p$ is a necessary condition of $q$.

A new road link is a necessary condition for a change in the spatial configuration system.

An essential positivist explanatory model requires sufficient conditions for explaining the relationship between cause and effect. Thus, we are interested in the sufficient conditions to explain a certain phenomenon in street network research. In our example, a new road link effectively triggers changes in a given configuration of a spatial system. Likewise, other conditions (e.g. road blockages or walls with closed gates) can result in a configurational spatial change. However, it is sufficient that only one of them comes into being for bringing about changes in a city's spatial configuration system. In essence, research on street networks sets out conditions sufficient for a change in the built environment. In a more refined perspective, von Wright introduces explanatory models as to why something was or became necessary, or, conversely, why something was or became possible. In the "why necessary" type of explanations, sufficient conditions are crucial, and in the "why possible" type, necessary conditions are crucial (von Wright 1971, p. 58). Below, both explanatory models are presented using an example.

Positivism explanatory model

Why something became necessary: A new road link is a sufficient condition for spatial configurable change.

Hermeneutic explanatory model

Why something became possible: A new road link is a necessary condition for spatial configurable change.

The second example appears to be inadequate because necessary conditions are redundant when dealing with cause and effect relations. In essence, space syntax focuses on sufficient conditions of changes in urban space using a positivism approach when interpreting the results from the axial and segment analyses. Any kind of change in the urban space is sufficient in such a way as the integration values will change. In line with the positivistic tradition, we focus on the city as an object, free from any meanings, intentions, and memories attached to it. Therefore, the sufficient conditions explain a phenomenon, which is related to cause and effect and not to the intentions behind a cause.

Counterfactual considerations are another means to consider the relationship between cause and effect. Applying counterfactuals is useful to set out what happens if a prediction is not verified. For example, if a new road link were not constructed, no change in the spatial configuration system of an urban area would occur. Conditions of this form are relevant, and no spatial change in an urban street network implies no changes in the dispersal of integration values in a city.

\subsection{Space Syntax's Hermeneutic Explanatory Models}

As space syntax research has shown, high spatial integration of the street network implies large numbers of people in the streets (Hillier et al. 1993, 1998), high levels of various economic activities (Van Nes 2002), high property prices (Desyllas 2000), high building density, and a high degree of multi-functionality (Ye and Van Nes 2014). We have so far discussed this independent from the influence of different cultures, societal groups, identities, and beliefs, and here we will set out how space connected to a social rationality belongs to a hermeneutic tradition. Social rationality deals with situations in which not all alternatives, consequences, and event probabilities can be foreseen. Research on space and social rationality regarding 
present or past issues mostly presents explanations embedded in meaningful reasons and consequences. Although causes occur before effects, it is often easier to identify and observe effects. Thus, explanations often set out from the effects and seek to discover their causes. Again, we apply von Wright's logic for our example:

Effect (= consequence): Clashes between various ethnic and religious groups decrease when these groups are located in neighbourhoods that are physically separated from each other.

Cause (= reason): Walls contribute to separating various neighbourhoods from each other.

Of course, this causal explanation is too general and is not uniformly valid. We need context-dependent cases. For example, in the case of Belfast's peace lines, the walls used as separation barriers contributed to reducing clashes between Protestants and Catholics. For the Berlin case, the Berlin Wall separated East and West Germany and aimed to prohibit East German citizens from fleeing to West Berlin. On the other hand, in the case of Tel Aviv and Jaffa, the 'wall' was only on paper, thus a mental boundary, but it had the same effect as a physical wall and Jews and Arabs avoided mingling with each other (Aleksandrowicz et al. 2018). Research concerning social rationality is context-dependent. In order to explain the occurrence of a particular phenomenon, reference to concrete circumstances is mandatory. The case of Jaffa and Tel Aviv's 'paper' wall presents this as follows:

Effect (= consequence): The clashes between Arabs and Jews are reduced in 1944, in Jaffa/Tel Aviv.

Cause (= reason): The administratively planned borders separate the Arab neighbourhoods from the Jewish neighbourhoods.

When constructing a new suburb with the idea of separating Arabs and Jews, it is difficult to derive from its implementation how its residents will behave in the future. It is also difficult to propose any kind of regularity or predictability with regards to the influence of this particular neighbourhood on other urban areas. Moreover, research in the field of space and social rationality requires studying the past in order to gain an understanding of intentions, which lead, for example, to conflicts or to anti-social behaviour. Interpretations concerning meaning, purpose, and behaviour are ensured from their contextualised appearance, but it seems difficult to assess the explanatory power of cases involving human intentions with causal explanatory models. This kind of research seeks to understand the intentions behind certain behaviours or cultures rather than explaining causes. Subsequently, in research traditions belonging to the human and social sciences, the aim is to understand the reasons, intentions, and motives associated with these causes. Therefore, explanations for forthcoming effects like future urban developments or certain behaviours are not provided. Accordingly, research on space and social rationality can only indicate that certain changes will occur, but not exactly how they will occur.

For the research area of space and social rationality, it seems appropriate to present von Wright's concept of intentionality and his model of teleological explanation. It might seem impossible to invest such a model with a certain degree of predictability using our approach; however, a key method for analysing its explanatory power might result from an analysis of the meaning of the explanandum (Fig. 6.8) or the phenomenon that has to be understood (von Wright 1971, p. 135).
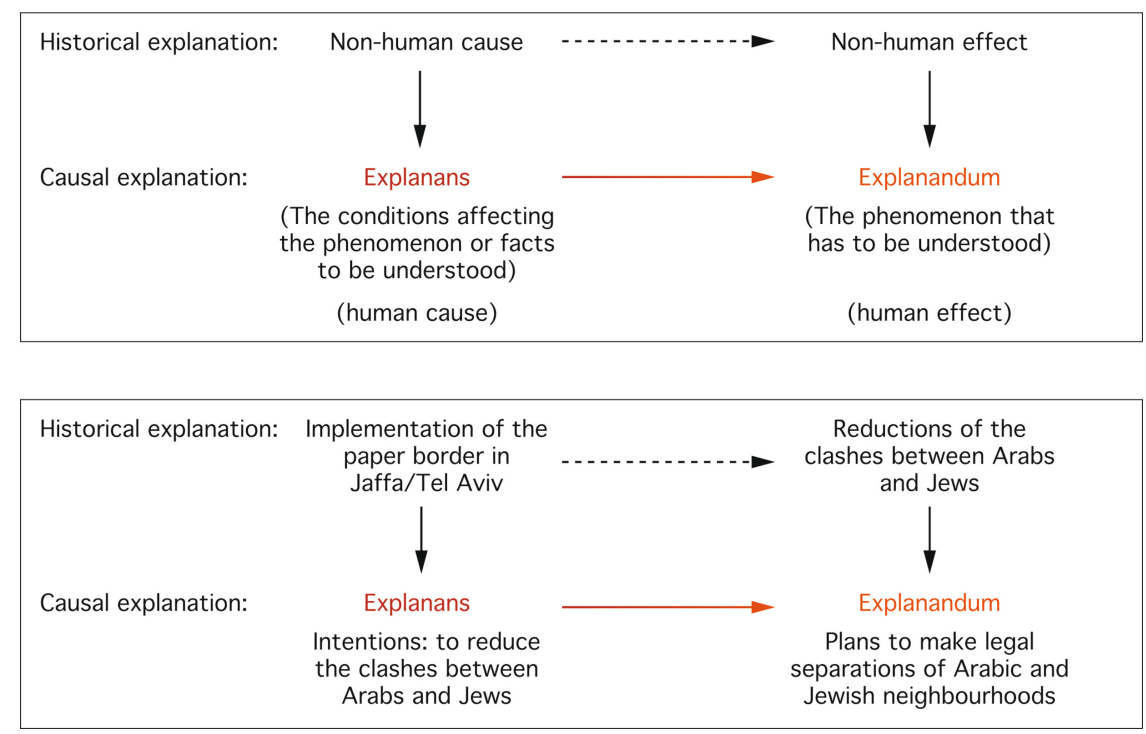

Fig. 6.8 An example of teleological explanations using the Jaffa-Tel Aviv 1944 case 
In the Jaffa-Tel Aviv case, when knowing the intentions that brought about blocking the inter-accessibility of the street networks, it becomes easier to account for its effects even it is not possible to predict them on behalf of the intentions. Acknowledging that a historical approach is needed for understanding intentions and behaviour implies that research on space and social rationality can only examine individual cases and cannot outline a 'theory' of human intentions.

Research related to cultural, historical, and social issues is embedded in already existing urban areas. However, it is impossible to predict in a positivistic manner how newly constructed neighbourhoods will function socially. The explanatory power of research on space and social behaviour depends both on human factors like intentions and purposes and on the physical layout of a built environment.

Human intentions or purposes and actual incidents often influence each other. Their nested interactions reiterate. Von Wright illustrates 'quasi-causal' historical explanations of this sort by the model presented in Fig. 6.9 above (von Wright 1971, p. 143) using a context-dependent example. The whole explanation process is split into sub-reasonings, and one explanation and understanding leads to the next until the explanandum is reached. Each sub-result in the process results in a new situation and allows for new intentions and purposes.
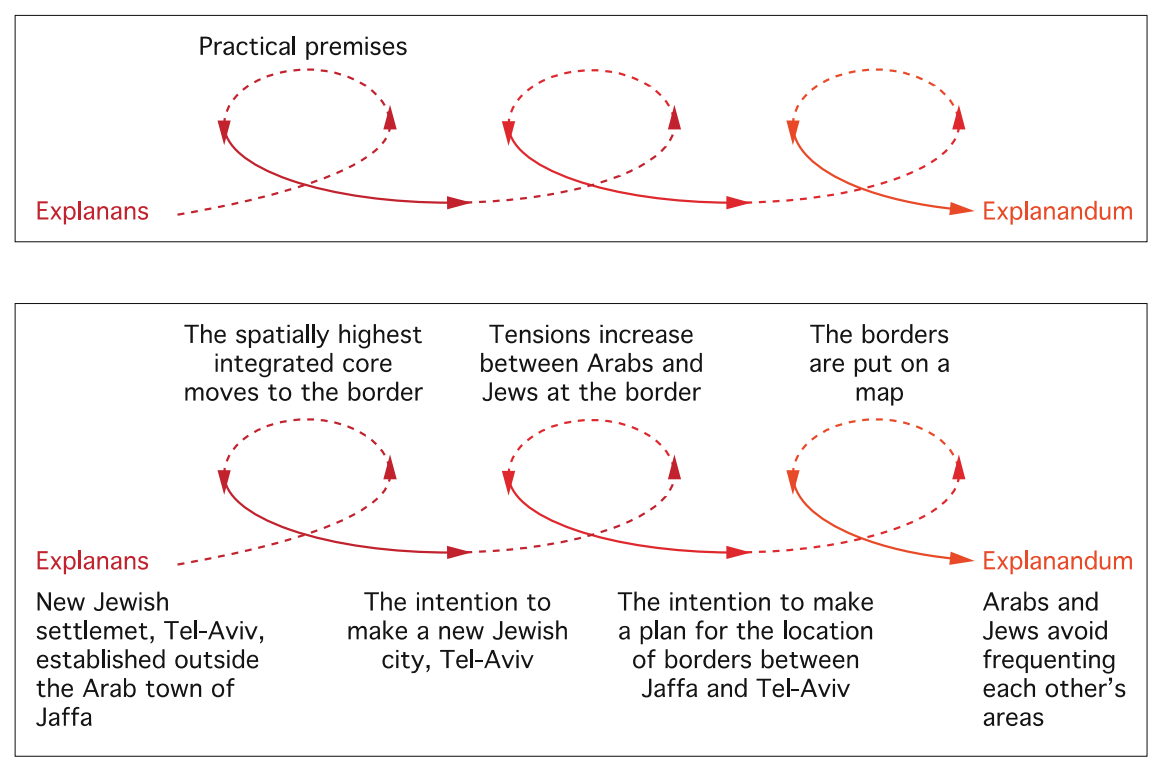

Fig. 6.9 An instance of a 'quasi-causal' historical explanation

Using this model for our example about space and religious conflict is appropriate when an understanding of meaning and intention beyond the implementation of walls in neighbourhoods is at stake. True understanding requires asking what was necessary for something to become possible (von Wright 1971, p. 154). Historical incidents allow for further developments because they transform intentions into effective action, especially by introducing new aspects with new possibilities to influence the course of outcomes (von Wright 1971, p. 155).

Historical explanations are mainly concerned with the necessary conditions for how something became possible (von Wright 1971, p. 136). Causal explanations, searching for sufficient conditions, do not pertain to research in history or sociology. In the following, we will show how von Wright's explanatory model, including necessary conditions, applies to research on space and social rationality. Because the research of this kind pertains to context-dependent situations, the following disposition parallels these schemata with a particular instance:

Hermeneutic explanatory model

$p$ is a necessary condition of $q$.

The intention for the Jewish immigrants to build their ideal city was a necessary condition for marking the administrative borders on the map in Jaffa city.

Research on space and social rationality is supposed to reconstruct the past, and therefore, has to search for the main intention as a necessary condition for the development of a certain kind of urban area. In what way the spatial layout of a neighbourhood influences the social behaviour of its users is too complex to be derived and depends on extra-intentional 
factors. One cannot predict whether a certain behaviour will occur, nor can one assess in what way a certain behaviour will take place. The explanatory power of research on space and social rationality consists only in the identification of the intentions that are necessary for a given course of events.

Our examples for explanations show that research on space and social rationality does not lead to general statements on built environments due to their context dependency. Grasping the sense of a neighbourhood's spatial layout depends on an understanding of the cultural preconditions producing the socio-spatial configuration. A society's ideologies, symbolic values, and attitudes are under constant change, and their articulations vary from one settlement to another. An interpretation of how activities in a society shape urban space thus requires an understanding of both the built environment itself and its position in its comprehensive context.

Interpretation processes that account for such interactions between parts and wholes of physical objects and their meanings are often called hermeneutic circles (Føllesdal et al. 1996, p. 105). For our research on space and social rationality presented here, this form of scrutiny consists of at least the following phases:

(1) Identifying various spatial layouts

(2) Understanding them first in terms of the intentions that necessarily conditioned their existence

(3) Destroying or altering the achieved understandings of built form in terms of the intentions they acquired.

(4) Assessing their relationships with the built environment in its entirety.

Any progress in one of these phases leads to a refined or revised representation of their meaningful existence in subsequent above-mentioned phases. The usefulness of the hermeneutic approach contributes to increased awareness and understanding of cultures and the meanings attached to physical objects and spaces for particular contexts. Here the results from the space syntax analysis are used for understanding the spatial features of particular cultures or the occurrence of a certain kind of human behaviour.

For gaining understandings and making theories on how cities work, there are some simple rules to follow. First, when dealing only with the physical part of the built environment free from human intentions, meanings, and memories, positivistic explanatory models are used. Here explanations and theories can be made based on various spatial combinatorics (demonstrated in Sect. 6.3). When blocking a street, making a new street link, etc. the various spatial integration variables for all streets will be affected, but to different degrees. One physical change is a sufficient condition for making something necessary. Research of this kind is context-independent and contributes to general explanations and universal theories. These theories can explain the future effects of present spatial changes and can explain the spatial set-up of the space syntax analyses of excavated towns. Second, when dealing with human intentions, meanings, and memories, hermeneutic explanatory models are used. Here we are dealing with gaining an understanding of a particular phenomenon taking place. Space syntax can only describe the spatial structure and cannot derive future effects from a particular case. Often, research of this kind seeks to identify the necessary condition as to why something became possible for each particular case. Therefore, space syntax research of this kind is heavily context-dependent and has a low degree of predictability and generality. Third, when dealing with human intentions that are unambiguous, positivistic explanatory models can be used. Market rationality (profit maximising) and time efficiency are unambiguous intentions. Therefore, it is possible to predict future impacts on economic-related activities and movement flows as an effect of spatial changes to the mobility network.

\subsection{Conjunctions and Disjunctions of a Phenomenon}

What happens when several causes have to be present so that a given effect can take place? When using more than one condition in the explanatory models, the type of condition changes with regards to positivism and hermeneutics. Positivistic explanatory models deal with sufficient conditions for only one condition and deal with a complex set of necessary conditions when dealing with several conditions in one statement. Conversely, the hermeneutic explanatory models deal with necessary conditions for only one condition and deal with complex sets of sufficient conditions when dealing with several conditions in one statement. This difference in whether we are dealing with necessary or sufficient conditions requires us to focus on whether we are dealing with one or several conditions.

A complex sufficient condition consists of conjunction of states of affairs (von Wright 1971, p. 39). Given the following example: 


\section{Hermeneutic explanatory model}

Maybe $p$ or $r$ alone is sufficient for $q$ to occur. But if $p$ and $r$ occur together, $q$ is certain to occur too.

There can be a whole list of conditions, but all of them have to be present for the given effect to take place. Here is an example from space syntax research from Rotterdam (Van Nes and Rooij 2015):

\section{Hermeneutic explanatory model}

Maybe a highly integrated street network or intervisible streets alone is sufficient so that the presence of women in Rotterdam's streets will occur. But if a highly integrated street network and intervisible streets occur together, the presence of women in Rotterdam's streets is certain to occur.

This example with a set of complex sufficient conditions pertains to research belonging under a hermeneutic research tradition. All the conditions have to be identified and present for the effect to occur. Moreover, context-dependent situations are required.

Often, these kinds of explanatory models with complex sufficient conditions are used for identifying all of the spatial parameters when describing specific neighbourhoods with social problems. Conversely, in a complex necessary condition $p$ and $r$ are logically separated from one another. While a complex sufficient condition consists of conjunction with a phenomenon, a complex necessary condition presents itself as a disjunction. The following example shows how space syntax analysis on only the physical part of the built environment accounts for complex necessary conditions:

\section{Positivistic explanatory model}

Maybe $r$ does not require the presence of $p$ (unconditionally), nor the presence of $q$ (unconditionally); but $r$ may nevertheless require that at least one of the two, $p$ or $q$, be present.

\section{Positivistic explanatory model}

Maybe spatially configurable change of the street network does not require the presence of a new road link (unconditionally), nor the presence of a road blockage (unconditionally); but spatially configurable change of the street network may nevertheless require that at least one of the two, a new road link or a road blockage, be present.

However, for research on spatial combinatorics, sufficient conditions or complex necessary conditions are at stake. Here is one example with the use of the complex necessary conditions. We illustrate it with an example from space syntax research.

\section{Positivistic explanatory model}

Maybe $r$ does not require the presence of $p$ (unconditionally), nor the presence of $q$ (unconditionally); but $r$ may nevertheless require that at least one of the two, $p$ or $q$, be present.

\section{Positivistic explanatory model: Theory of spatial combinatorics}

Maybe increased depth does not require the presence of a centrally placed object (unconditionally), nor the presence of continuous blocks (unconditionally); but increased depth may nevertheless require that at least one of the two, a centrally placed object or continuous blocks, be present.

Therefore, complex necessary conditions pertain to research belonging under a positivistic research tradition. Herein cause and effect relations are explained and theories are built that account for all kinds of built environments. In the following sections, we will reflect upon what space syntax has contributed to theory building and to our understanding of how built environments work.

\subsection{Research Connected to Space Syntax in the Positivism and Hermeneutic Traditions}

In the following sub-chapters, we will describe how space syntax has contributed to understandings and explanations of urban space and social, as well as economic-related activities. Before going further, we repeat the following before discussing what parts of space syntax research belong under a hermeneutic or positivistic approach:

(1) Positivism: uses sufficient conditions when there is only one condition in an explanation, and a complex set of necessary conditions when there are several conditions present in an explanation. Positivistic explanatory models are used when dealing with objects free from human intentions, meanings, and memories and with unambiguous human intentions such as market rationality and time efficiency. 
(2) Hermeneutics: uses necessary conditions when there is only one condition in an explanation, and a complex set of sufficient conditions when there are several conditions present in an explanation. Hermeneutic explanatory models are used when dealing with objects with human intentions, meanings, and memories and to understand the local social and cultural context.

\subsubsection{Three Spatial Parameters for Urban Centrality}

For an urban street network, integration analysis depicts the location of vital urban centres. Urban centres are located along the highest spatially integrated streets, and if the spatial configuration of the street structure changes, the location of urban centres might also change.

Hillier $(2009,2012)$ refined the concept of centrality for cities as pervasive centrality, whereby centrality functions diffusely throughout the network at all scales (including closeness and proximity to smaller and much larger centres from a certain location within the city). In this context, space syntax makes a link between emergent structure and spatial agency, where a city's emergent structure is a law-governed process of the network of spaces that links buildings together (the morphological component). In turn, the spatial agency in itself has effects on the functional patterns of the city or the behaviour of the people. At first sight, centrality seems to be clear and stable, and the central area and its boundaries seem well defined. All we have to do is to reveal the spatio-economic layout. However, as soon as we take temporal aspects into account, the idea of stable and clear centres seems to fade, and they shift, expand, shrink, or change their focus (Yamu 2014, 2020). The location of urban centres depends on how the spatial structure of the street and road network changes over time.

Space syntax is able to quantify the spatial properties between urban blocks in at least three ways connected to centrality, namely metric distance, topological distance, and geometric distance. The question now is how these various types of measures contribute to knowledge on urban centrality.

\section{Metric centrality}

Metric centrality implies that an urban centre is located in the middle of a built environment with the shortest metric distance to all other points in that area. This measurement is free from temporal aspects such as travel time and aspects related to travel time such as congested junctions, traffic lights, complex roundabouts, and bad street or road surface quality. To be located metrically in the middle of a settlement does not always mean having the most inter-accessible location.

\section{Topological centrality}

Topological centrality deals with the spatial configuration of the street and road network in terms of the number of direction changes. A centre that is located topologically in the middle of a built environment has the fewest direction changes to all other streets in comparison with any other streets of that built environment. The more broken up a street network is in a built environment, the weaker the spatial conditions become for generating a vital economic centre. Topological centrality is about the degree of accessibility in terms of the fewest direction changes. Urban areas with a high degree of topological centrality have the highest degree of to-movement potentials. In these areas, shops tend to cluster and the rental and property prices tend to be higher than other areas in the same city.

\section{Geometric centrality}

Geometric centrality deals with changes in angular directions when moving from anywhere to anywhere else. A centre that is located geometrically in the middle of a built environment has the fewest angular deviations to all other streets in comparison with any other streets of that built environment. Geometric centrality is about how the main route network links a city's edges and local areas towards its centre through the least deviation in angular direction. Urban areas with a high degree of geometric centrality have the highest degree of through-movement potentials. These streets tend to have the highest flow of movement. Shops tend to locate themselves along these streets in order to catch the random through travellers.

Metric, topological, and geometric centralities concern the spatial issues of a built environment. Other concepts of centrality are required to describe the social and economic activities taking place inside urban centres. In general, there is a difference between economic and cultural centrality. 


\subsubsection{Economic and Cultural Aspects Related to Urban Centrality}

An economic centre is defined as the place where trade, shopping, and finances take place. The aim for these kinds of activities is to be both in a central metric, geometrical, and topological position to all potential customers. This phenomenon can be explained with positivistic explanatory models because we are dealing with market rationality where the human intentions are unambiguous. Here we are dealing with sufficient conditions and complex sets of necessary conditions.

As research has shown, the optimal position of an economic centre depends on the structure of the street network (Bruyns et al. 2007, p. 35). The spatial configuration of an urban street network on a micro and macro scale seems to determine the distribution of shops. A successful shopping area has both a globally and locally strategic position in a built environment (Hillier 1999b, p. 119). Not only the most integrated streets on a local and global scale decide where shops locate themselves, but also a shopping street's degree of connectivity to its direct side streets. If an economic centre's optimal position changes through changes in a street network, the location of this centre is likely to change too (Hillier 1999b, p. 110ff; Van Nes 2002 p. 287ff). Likewise, changes to the street network affect the flow of human movement. These processes occur in a context free from a state-controlled economy, regulations on land use, and political, religious, or organisational constraints.

Berlin's old and new high street Friederichstraße, discussed in Chap. 2, provides an example of how changes in the street network configuration affect the location pattern of shops. At present, this street is undergoing the multiple effect process where it is slowly becoming the high street of the newly reunited Berlin. First Friederichstraße became a well-used route after the removal of the Berlin Wall in 1989. Then the first shops established themselves, which attracted more pedestrians, which in turn, led to more new shops along this street.

Economic centrality is not something static, and it is a dynamic process. "At all levels of the hierarchy, centres grow and fade, often in response to changing conditions quite remote from the actual centres" (Hillier 1999b, p. 108). Therefore, an economic centre is heavily dependent on a street structure that relates to both topological and geometric centrality. Accessibility to potential customers is the main issue, and thus high spatial integration of the street network is a sufficient condition for economic centrality.

The way human beings act and behave in urban spaces depends on their motives and intentions. In most cases, the underlying intentions behind human being's actions are difficult to predict. Therefore, their behaviour after urban interventions can also be difficult to predict. With regards to market rationality, the intentions seem to be unambiguous because they concern profit maximising. Therefore, it is possible to predict where economically motivated activities will take place because they have to be in an optimal location in order to survive in a competitive market (Van Nes and Yamu 2018).

To date, research has shown that the location of shops, retail outlets, and national and international firms and the location of urban centres depends on a high degree of integration of the street network on various scales (Hillier 1999b; Hillier et al. 1993, 1998; Rocco and Van Nes 2005; Van Nes 2005; Mohammed and Van Nes 2015; and Van Nes 2002). Likewise, land values and rents tend to be influenced by the various integration values of the street network (Desyllas 2000). High degrees of accessibility, visibility, adjacency, permeability, and reachability are all complex necessary conditions for creating vital urban centres and for generating economic activities within them.

Figure 6.10 shows space syntax analyses of Coventry from 1940 and 1999. In the 1960s and 1970s, large changes to the street and road network were made, and these affected the degree of spatial integration of the town centre. Before WWII, Coventry had a highly integrated and vibrant pedestrian-based town centre. After the implementation of the inner ring road, the town centre consisted mostly of a large shopping centre accessible from the ring road. The intention of the ring road was to reveal the town centre to through traffic. However, the effects of the ring road were that the streets inside Coventry's shopping centre were 'dead' after opening hours (Van Nes 2021). Obviously, changes in the street network can affect the location of the economic centres in the built environment. 


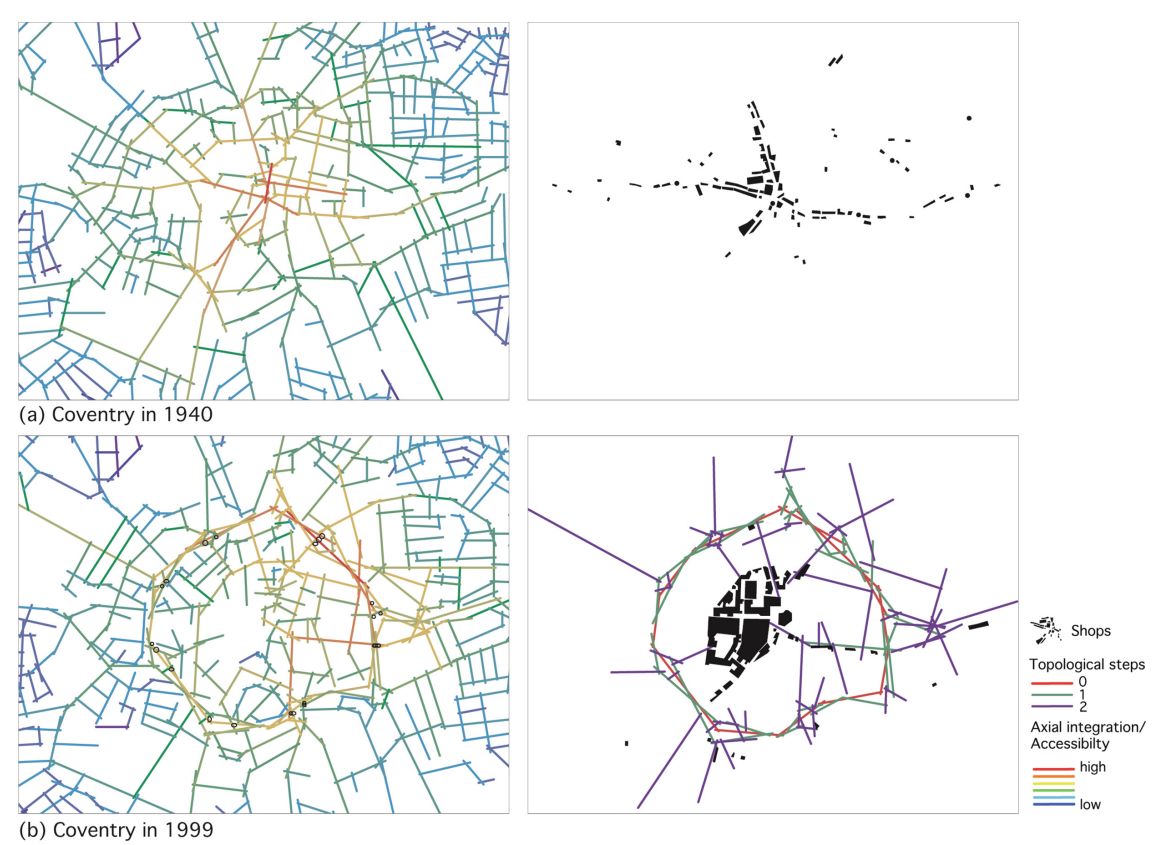

Fig. 6.10 Global integration analyses of Coventry before (a) and after (below) implementation of a ring road with the location pattern of shops (b) depicting the shift of centrality over time

As research has shown, land use development and building densities adjust themselves according to the degree of street network integration in built environments in a natural urban transformation process. If an urban area has low spatial integration of its street and road network or is poorly connected to its surroundings, the area tends to be mono-functional, have imbalances in the FSI and GSI of the built mass, and lack street life and diversity of economic activities. Conversely, an urban area with high spatial integration of its street and road network tends to be multi-functional with high density of the built mass and having a vibrant street life (Ye and Van Nes 2014; Van Nes et al. 2012). Therefore, the spatial structure of the street and road networks matter in how urban areas transform over time.

Figure 6.11 shows four different urban transformation stages of four Dutch towns, all of which have around 100,000200,000 inhabitants. The first settlements of the planned new towns of Lelystad and Almere took place in the 1970s. Zoetermeer was a small village that expanded to a large town in the 1960s and 1970s, whereas Haarlem is an old city dating back to the Middle Ages. The areas classified as suburban and low urban have low spatial integration of the street network on all scale levels, have low building density, and are mono-functional urban areas. Conversely, the middle and highly urban areas have high scores on all spatial parameters. The urban areas labelled as 'in-between' are under different stages of urban transformation. In most cases, the degree of street network integration has middle or high values, whereas the degree of building density and land use diversity has low or middle values.

These findings can furthermore be connected to the theory of natural urban transformation process. This theory is inspired by a common understanding that roads exist on average for thousands of years, a building typically stands for about 100 years, and the functions inside buildings tend to change frequently (Van Nes 2002). This theory can be verified by selecting a set of new and old towns with similar sizes and regional positions but belonging to different urban transformation phases from newly built to long-settled. The application of space syntax, MXI, and Spacematrix methods on Norwegian (De Koning et al. 2017), Dutch, and Chinese cases (Ye and Van Nes 2014) showed how street network integration, building density and typology, and degree of land-use mixture interact with each another in different phases of an urban transformation process.

There are some aspects of urban centrality that touch on the limits of space syntax. Studies on built form and meaning also deal with place character and architectural styles. Places with a large concentration of historically important buildings and monuments from the past are defined as cultural centres. The artefacts' meanings and the traditions related to them can be understood from the technical, social, cultural, and economic activities that took place in the past (Rossi 1983; Moudon 1997). Thus, studies on cultural centrality have to be approached from multiple angles. Here we are oscillating between a hermeneutic as well as a positivistic approach. 


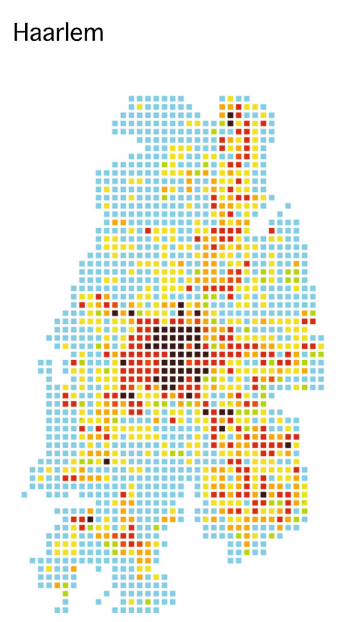

Zoetermeer

Suburban

Low urban

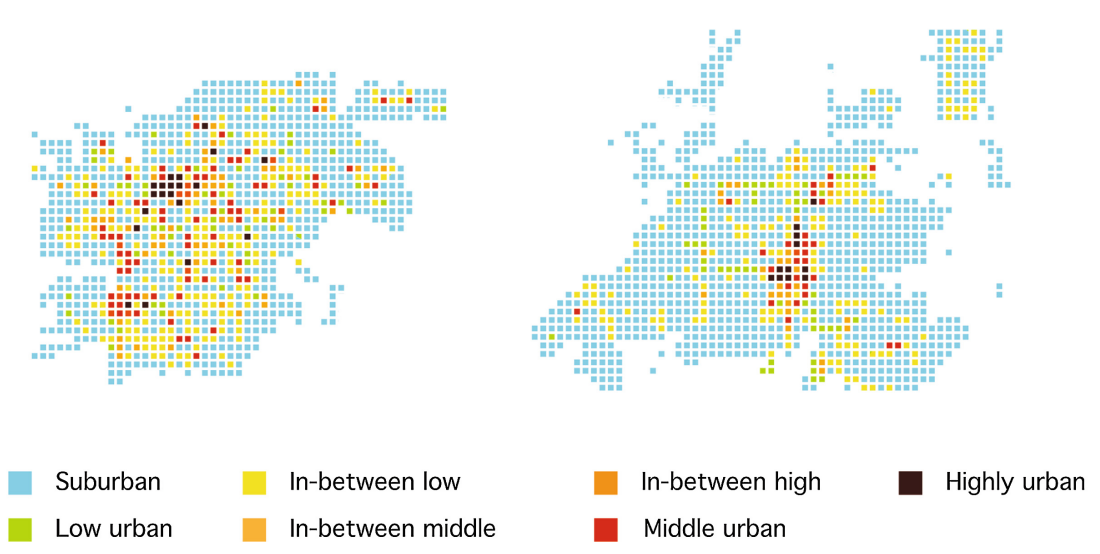

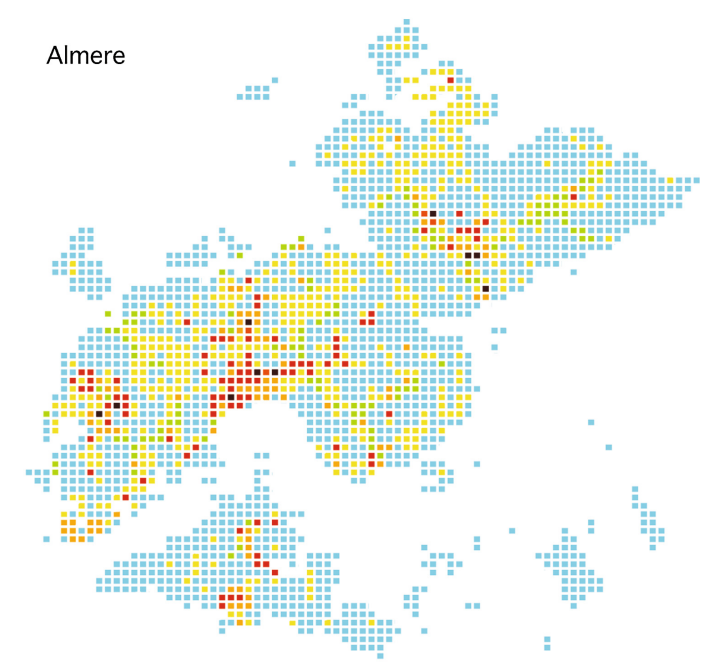

Lelystad

Fig. 6.11 Correlations between space syntax, MXI, and Spacematrix for three new Dutch towns and one old Dutch town (Ye and Van Nes 2014)

Cultural centrality can be multifaceted and can include, for example, religious, spiritual, trading, political, administration, or education centres. Many old European cities have a large and intact historical city centre with several significant artefacts with attached collective memories. In the past, many of these centres had a strong position in terms of politics, trade, and cultural activities. These activities from the past mostly have left their mark in monuments, for example, sets of buildings and street patterns. The establishment of cultural centres often occurred along spatially highly integrated street networks in the past. Later on, through city growth, many of these cultural centres lost their spatially central positions. However, intact historical urban centres function as attractors for cultural activities such as museums, arts and crafts, tourism, concerts, workshops, performances, etc. All of these activities and their artefacts seem to create the atmosphere and sense of a particular place (Rossi 1983; Norberg-Schulz 1980,1971). These kinds of places tend to become attractors for tourism, even though these places have lost their spatially central positions through urban growth and changes in the overall street network structure.

Figure 6.12 shows examples of cultural centrality and economic centrality. Amsterdam's central square (the Dam) is an example of cultural centrality. As the city grew, its topological and geometrical position changed. However, the centre has a high concentration of historically significant buildings with high architectural qualities, intact streetscapes and channels, and artefacts with high levels of collective memories. Conversely, the ZuidAs in Amsterdam is an example of pure economic centrality. After the implementation of Amsterdam's ring road, the area became the most spatially central location in Amsterdam as well as in the whole Randstad. The land along the southern part of Amsterdam's ring road attracted various advanced product and service companies along with foreign directed investment companies. Since the 1990s, the area has changed from a more or less no-man's land into the main financial and business centre of the Netherlands. 
Fig. 6.12 Example of economic centrality (the business district at the ZuidAs in Amsterdam) and cultural centrality (Dam square in Amsterdam's historic centre)
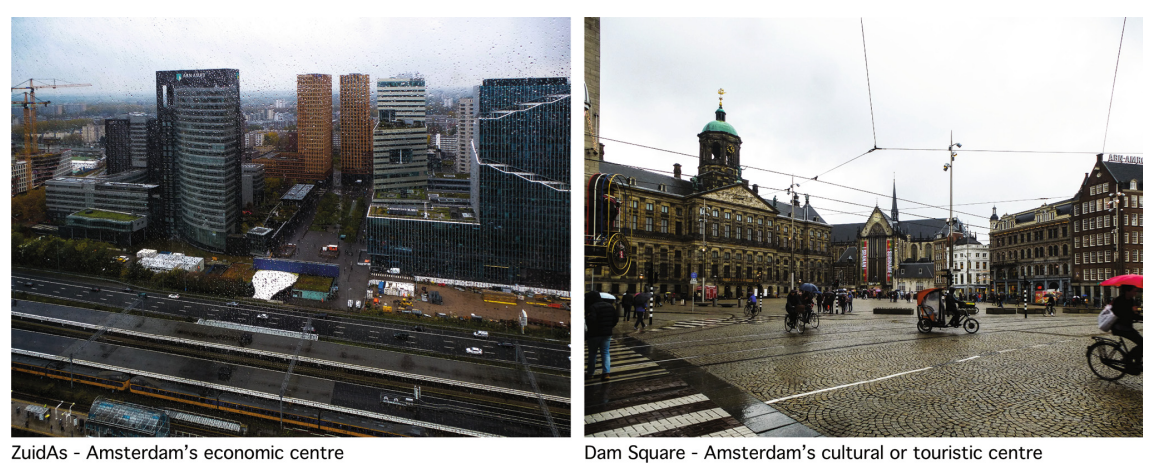

Space syntax does not analyse place character or spatial order, but spatial structure. The method is able to analyse the spatial configuration of past street patterns from old maps and to correlate the results with the location of remaining significant old buildings. Often the locations of these kinds of buildings are determined by strong economic centres, with their accordingly highly integrated street networks from the past. Therefore, the application of space syntax on old maps provides explanations or understandings as to why these centres were highly vital in the past in comparison with the present situation. Cultural centrality implies an understanding of cultures, built forms, and meanings based on past human intentions. Therefore, there exist no theories on these issues. Instead, we have to deal with a context-dependent social rationality and the intentions behind a cause, which will be discussed in the next section.

\subsubsection{Dealing with Understandings: Context-Dependent Space Syntax Research in Line with the Hermeneutic Tradition}

What has space syntax contributed to the understanding of the social effects of spatial segregation? In general, segregated streets have more complex routes to all other streets in a built environment, and these spatial features can affect how human beings perceive, behave, and orientate in built environments. In contrast with market rationality, where the intentions tend to be unambiguous, social rationality concerns a wide range of rationales with a wide range of intentions. In the following sections, we reveal the explanatory power of some results from space syntax research where theory building is not an issue due to context dependency. Here we are limited to only gaining understandings about a phenomenon. As mentioned earlier, we are dealing here with necessary conditions and a complex set of sufficient conditions.

\subsubsection{Space and Crime}

In research on urban space and human social interactions, it is difficult to set out general statements on how the spatial layout of a built environment can provoke criminal activities and anti-social behaviour and create social segregation. Even though incidents can be understood from a spatial point of view, knowledge of space and crime depends on understanding the behaviour of an area's inhabitants and having insight into the social composition of the residents. Sometimes the social composition of the inhabitants can overtake the spatial generative power. If a large number of social disadvantages are concentrated in a highly integrated residential area, this can lead to increased crime and vandalism (Rueb and Van Nes 2009).

An account of anti-social behaviour in terms of the extrinsic properties of space requires studying already established areas. It is about how a built environment's spatial organisation can disturb or encourage the natural relationship between residents and strangers.

Even though research on space and crime is heavily context-dependent, some common features are found in European built environments. Seemingly, neighbourhoods with street networks that are visually broken up and with few dwelling entrances constituting the streets are often affected by crime (Hillier and Shu 2000, p. 232; Hillier and Sahbaz 2005, p. 456; Van Nes and López 2010). The same investigations prove that spatial organisation can generate movement according to co-presence and co-awareness in built environments. Where the local integration of a street network is high and the number of people frequenting the streets is high, the lower the burglary risk tends to be in European cities. 
Causes for crime and anti-social behaviour in European cities seem to depend on at least the following spatial conditions:

- Poor correlations between connectivity and the local and global integration of the nearby environment.

- The segregated areas are many topological steps away from integrated streets.

- The spatial structure in the area is deep in relation to itself and in relation to the whole system.

- The spatial relationship between private and public space includes a large number of semi-private and semi-public spaces.

- There are a few doors and windows facing the street.

All of these spatial parameters generate spatially enclosed building blocks with high privacy, but without general social control and lacking natural co-presence and mutual surveillance (Hillier 1996, pp. 188, 194 and 201; and López and Van Nes 2010). The design of architectural space can thus affect the use of space, and this issue seems to touch upon the problem of architectural determinism. Architectural determinism claims that the built environment determines social behaviour, and therefore it can be possible to predict how an urban area will function after it is built. It is difficult to assess the question of to what extent a space syntax approach is a form of architectural determinism. Whether crime or social malaise will occur in spatially segregated areas or not naturally depends on the social compositions and behaviours of their inhabitants. Therefore, we have no 'theory on space and crime.'

A space syntax approach, on the other hand, can at least identify the spatial properties that can help us to understand why some already established urban areas have a high level of crime and social problems. These identified spatial properties provide precise understandings of how spatial configuration plays a part in broader social processes of perceived and actual decline. Thus, a space syntax approach can show that the spaces of a built environment can affect human behaviour (Hillier 1999a, p. 184ff). A space syntax approach contributes to an understanding that the means a built environment provides are physical, while its end results are functional — not visa-versa, as claimed in many writings on built environments.

Gaining understandings of space and crime requires an understanding of the local context. For each local context, the aim is to identify the necessary condition behind every cause for a chain of incidences. For understanding the intentions behind each separate cause, a complex set of sufficient conditions is needed for explaining the conjunction of the phenomenon space and crime of a particular place.

Fig. 6.13 Scatterplots showing the correlation between burglary risk and various spatial parameters in the Dutch towns of Gouda and Alkmaar
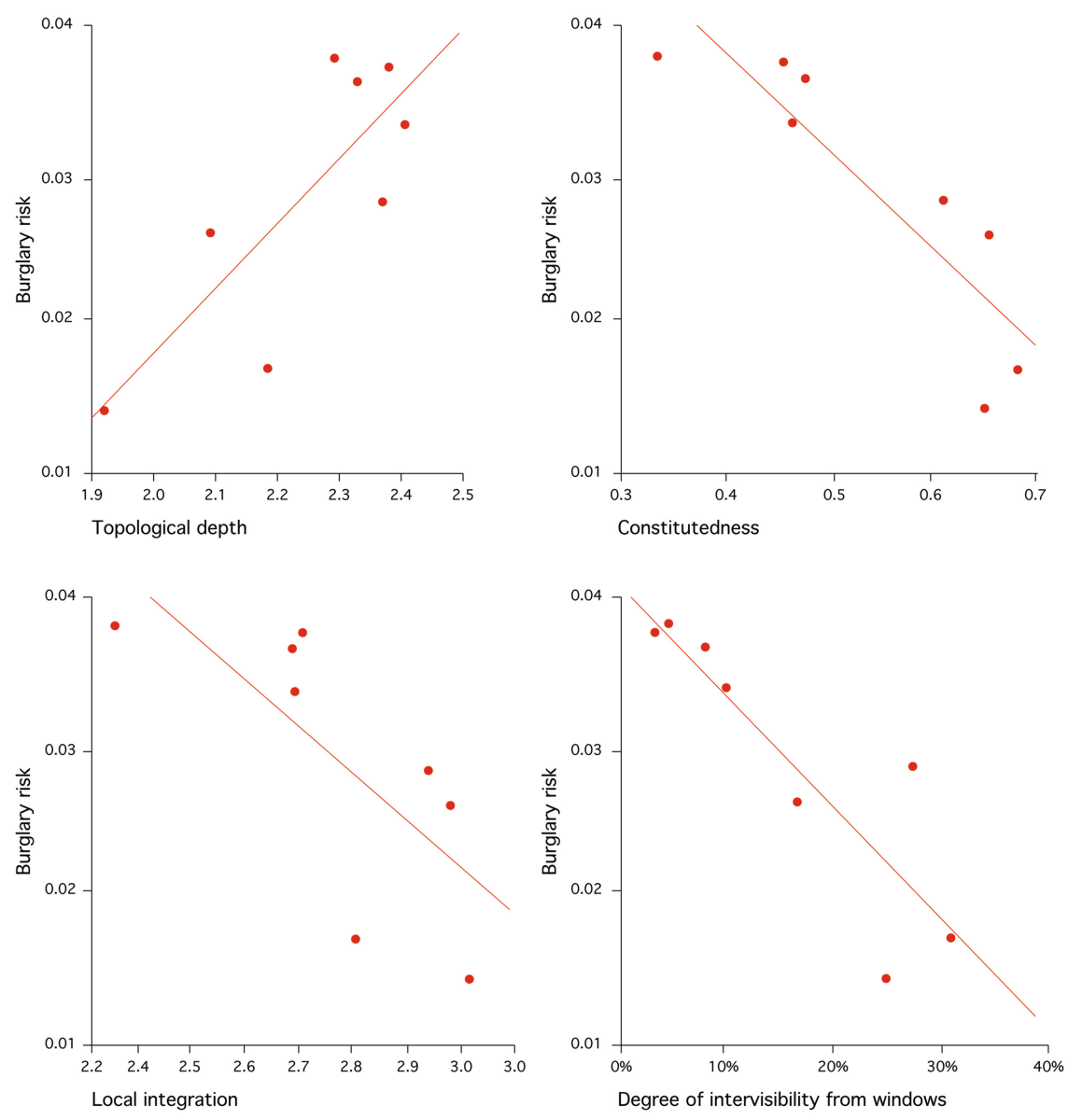
Hermeneutic explanatory model: the conjunction of phenomenon

Maybe $p, r, s$, or $t$ alone is sufficient for $q$ to occur. However, if $p, r, s$, and $t$ occur together, $q$ is certain to occur too.

Here we illustrate this with an example from research on space and crime in the Dutch towns of Gouda and Alkmaar (Van Nes and López 2010). We use the scatterplots in Fig. 6.13 as an example.

Hermeneutic explanatory model: The case of Gouda and Alkmaar

Perhaps the high number of direction changes (topological depth) from the main routes, the low local integration, the unconstituted streets, or the low intervisible streets was sufficient for the high burglary risk in Gouda and Alkmaar. However, when these four aspects occurred together, high burglary risk was sure to occur too.

\subsubsection{Space and Social Integration Versus Social Segregation}

As research has shown in the Netherlands (Van Nes and Aghabeick 2015) and Egypt (Mohammed et al. 2015), there is a correlation between the degree of spatial integration and the degree of social integration. Seemingly, spatial segregation contributes to social, as well as ethnic segregation among the users, while spatial integration supports the socio-economic integration of various social and ethnic groups.

A neighbourhood with low spatial integration of its street network on various scales and with a low degree of intervisibility of entrances and windows shapes the opportunities for social segregation. Segregated urban areas consist of a labyrinthine and broken up street network, dead-ends, and poorly intervisible streets. Social segregation occurs not only between various ethnic groups, but also between gender and age among the area's users. A neighbourhood with these spatial characteristics and with a large number of low-income non-western immigrants easily gains a reputation as a 'ghetto' (Van Nes and Aghabeick 2015). In contrast, when a neighbourhood with these spatial characteristics has a large number of high-income people, it easily gains a reputation as a 'gated community'.

Conversely, a neighbourhood with high spatial integration on the main routes going through the area, combined with all local residential streets being connected to the main routes and a high degree of intervisibility between entrances and windows on the ground floor level, generates social integration between various social and ethnic groups. Often visitors frequent the area due to a large number of exciting immigrant shops offering exotic products along spatially integrated main routes. A neighbourhood with these spatial characteristics and with a large number of non-western immigrants easily gains a reputation as a 'multicultural' neighbourhood (Van Nes and Aghabeick 2015).

A tree-structured street network with separate pedestrian movement routes contributes to spatially segregated residential areas with few opportunities for various types of people to interact. A network-structured street system contributes to the opposite. Hence, spatial segregation contributes to ethnic segregation. In spatially segregated areas, an emergence of a 'bubble within a bubble' takes place. The size of the personal space, or 'bubble', for various cultures plays a smaller role in spatially integrated main streets than in segregated streets (Van Nes and Aghabeick 2015).

Seemingly, the design and layout of the built environment affect how immigrants can integrate into the socio-economic life of the host country. There is a link between ethnicity, spatial behaviour, and spatial segregation. The degree of spatial integration influences the various ethnic groups' behaviours due to differences in cultural expressions of spatial behaviour and differences in cultural survival tactics.

Tearing down buildings and replacing them with new buildings or redistributing the inhabitants does not solve the problem of 'ghettos' on a longer term, and there is a need for understanding the relationship between spatial layout and the behaviour of various ethnic groups. Likewise, there is a need to understand which spatial parameters can generate socio-economic integration between various social groups. It can be possible to aggregate various vibrant multicultural urban areas where the immigrants shape their opportunities and interact with the culture and economy of the host country. "The mosaic of subcultures requires that hundreds of different cultures live, in their own way, at full intensity, next door to one another. But subcultures have their own ecology. They can only live at full intensity, unhampered by their neighbours, if they are physically separated by physical boundaries" (Alexander et al. 1977). A large variation of cultures contributes to making vibrant neighbourhoods. Seemingly, the underlying factor is the spatial layout of the neighbourhood that influences whether a neighbourhood gains a reputation as 'ghetto' or 'multicultural'.

Gaining understandings of social segregation and urban space requires insights into each particular culture of each social and ethnic group from a particular neighbourhood. For each local context, the necessary condition or complex set of 
sufficient conditions as to why something became possible needs to be identified. Here we illustrate a complex set of sufficient conditions for the Rotterdam case.

Hermeneutic explanatory model: the conjunction of phenomena

Maybe $p, r$, or $s$ alone is sufficient for $q$ to occur. However, if $p, r$, and s occur together, $\mathrm{q}$ is certain to occur too.

Hermeneutic explanatory model: The Rotterdam case.

Maybe spatial segregation, unconstituted streets, or a high number of low-skilled non-Western immigrants is sufficient for the neighbourhood in Rotterdam to gain a reputation as an urban ghetto. However, if spatial segregation, unconstituted streets, and low-skilled non-Western immigrants occur together, the reputation of the neighbourhood is certain to gain the reputation as an urban ghetto.

The same exercise can be done for a multicultural neighbourhood:

Maybe spatial integration, constituted streets, or a high number of low-skilled non-Western immigrants is sufficient for the neighbourhood in Rotterdam to gain a reputation as a multicultural neighbourhood. However, if spatial integration, constituted streets, and a high number of low-skilled non-Western immigrants occur together, the neighbourhood is certain to gain a reputation as a multicultural neighbourhood.

Figure 6.14 shows a small part of the registrations of various types of immigrants in the public spaces of a neighbourhood in Rotterdam. As can be seen, where the spatial integration of the street network is high, in combination with intervisible streets with a high density of entrances, there is also a high variation in the ethnicities of the people in the streets. As soon as the streets become segregated, combined with a low density of entrances, social segregation takes place.

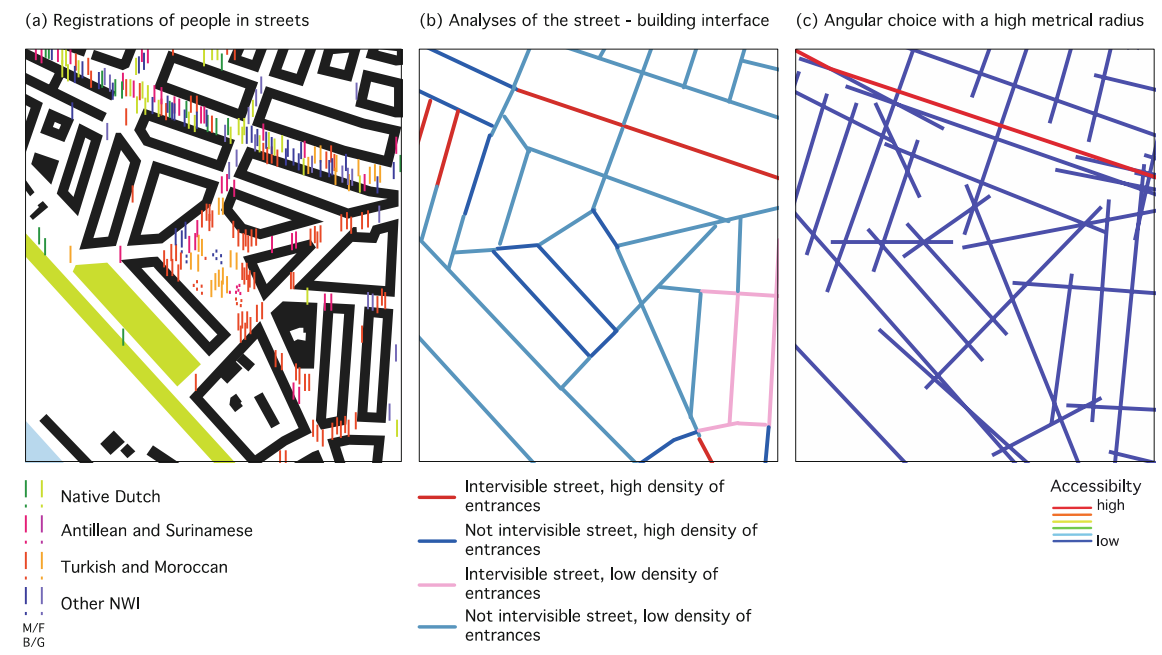

Fig. 6.14 Registrations of the ethnicities of people in public spaces (a) with various degrees of entrance densities and intervisibilities (b) and choice analysis with a high metric radius (c). Source (Van Nes and Aghabeick 2015)

\subsubsection{Space and Gender}

Research has shown that women and men use space differently in different cultures. However, a common feature is that women tend to avoid urban public spaces that are spatially segregated and that lack intervisibility and constitutedness (Nguyen and Van Nes 2014; Hardner et al. 2012; Van Nes and Rooij 2015).

Another study showed that neighbourhoods with poor connections between buildings and streets and with segregated streets generated social segregation among ethnicities, genders, and ages of users. Women were more frequently seen walking in vibrant, integrated streets and very rarely in the most segregated streets. Men dominated the segregated streets (Van Nes and Aghabeik 2015). Van Nes and Rooij's research in 2015 showed that vibrant streets were perceived to be safer and that the street network and micro-scale relationship between streets and buildings played a role in making such streets lively. Neighbourhoods lacking street network integration and intervisibility contributed to unsafe streets.

Research on preventing sexual harassment in built environments needs to add data on the degree of presence of women and men in the streets. Otherwise, the outcomes can lead to misguided strategies for improvement. Divergent strategies have been proposed for designing safe cities. Oscar Newman proposed in 1972 that spaces should be designed with large amounts 
of semi-public spaces separating inhabitants and visitors (Newman 1972). Conversely, Jane Jacobs proposed in 1960 that streets should be open for inhabitants and visitors at all times and that eyes from buildings on streets ensure natural surveillance. Maintaining urban safety is fundamental for vital street life, and only a few cases of violence in a street are enough to generate avoidance, thus contributing to unsafe streets (Jacobs 1960).

Two different scenarios for eliminating gender-based violence in streets can be imagined. The Newman-inspired scenario consists of urban areas for exclusively male or female use, and shopping, entertainment, education, and jobs are all clearly divided by the gender of their users. Clearly, no gender mixture in streets means no opportunities for sexual violence of men towards women. The Jacobs scenario presents a gender-mixed city where the vivacity of streets ensures their safety. In the latter, the seemingly utopian goal of ending sexual gender-based violence depends on not-entirely known factors of the physical environment and their relations with human behaviour, not to mention culture, education, and many other socio-economic aspects.

In a research project on space and sexual violence against women in Rotterdam from 2012 to 2017, correlations were found between the occurrence of sexual crimes, the amount of people and the amount of women in the streets, local integration, street function, and temporal aspects. Non-residential streets were safe during the day, but became dangerous during the night. Mixed land use was safer than mono-functional areas. A high degree of intervisibility of entrances generated a high degree of natural surveillance, resulting in greater safety in the streets. Residential streets with higher flows of people had fewer incidents than mono-functional commercial blocks, and commercial blocks had higher numbers of incidents after the shops closed due to the lack of surveillance (Miranda and Van Nes 2020).

As expected, women's avoidance strategies for crime prevention contributed to the reduction of incidents. However, they also contributed to a gendered space where the public urban spaces were not accessible for half of the population and led to misleading research results in which gated communities were considered to give the safest results. As recommendations for urban planning and policy practice, urban design including residences on both sides of streets and with good intervisibility between entrances on the ground floor level is needed. In commercial areas, diverse land use with dwellings and commercial functions is recommended (Miranda and Van Nes 2020).

Figure 6.15 shows some examples of 'gendered space' dominated by men and 'gender balanced' spaces in the Oud Mathenesse neighbourhood in Rotterdam. The typical gender-balanced streets are the shopping streets, whereas segregated and unconstituted streets tend to be male dominated (Van Nes and Rooij 2015).

Fig. 6.15 Examples of 'gendered spaces' dominated by men in images (a) and (b), and 'gender-balanced' spaces in images (c) and (d)
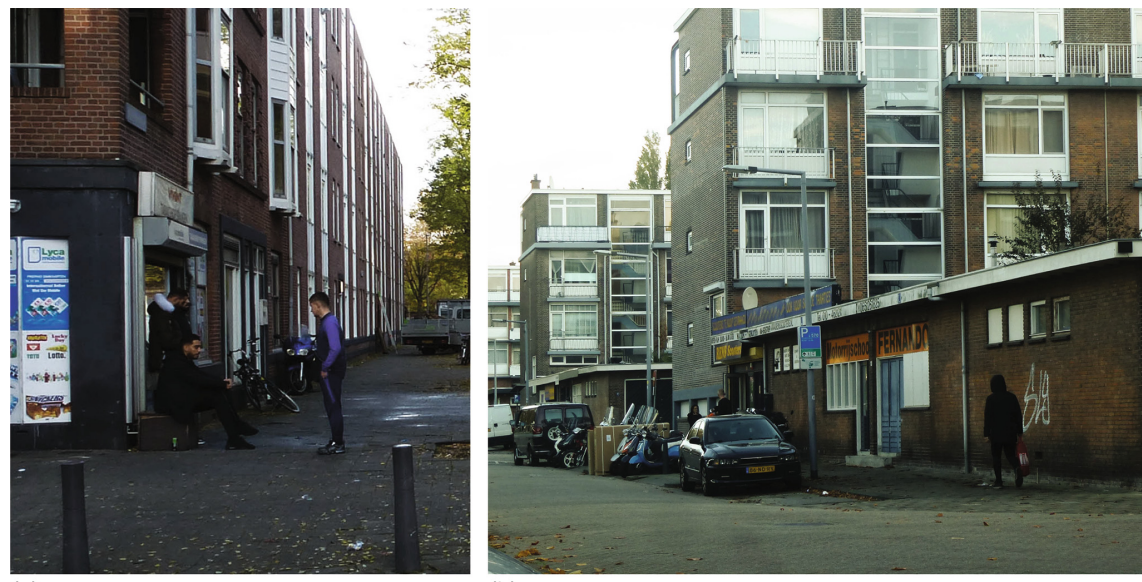

(a)

(b)

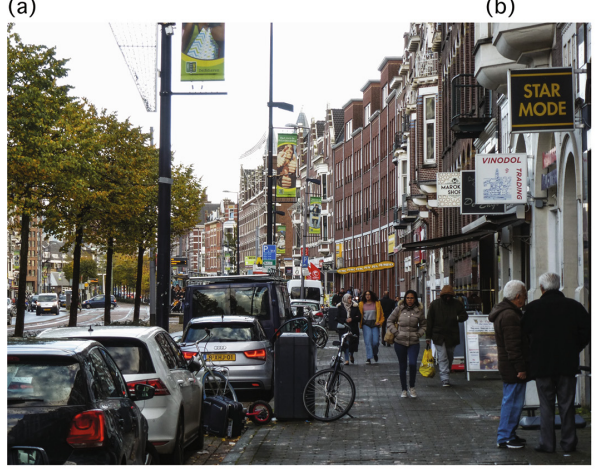

(c)

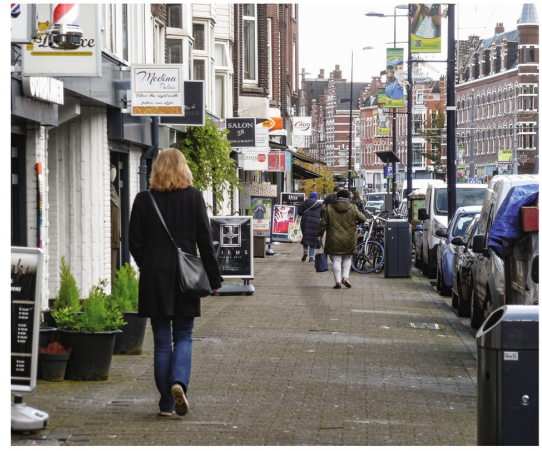

(d) 
Research on space and gender is context-dependent. The attitude towards women varies from culture to culture, and these affect the various behaviour patterns of women in urban spaces. A complex sufficient condition applied in the case above is as follows:

Hermeneutic explanatory model: the conjunction of phenomena

Maybe $p, r$, or $s$ alone is sufficient for $q$ to occur. However, if $p, r$, and $s$ occur together, $q$ is certain to occur too.

Hermeneutic explanatory model: The Spangen case in Rotterdam

Maybe an integrated street network, intervisible streets, or mix of active functions on the ground floor level is sufficient for gender-balanced street life in the Spangen neighbourhood in Rotterdam. However, if an integrated street network, intervisible streets, and mix of active functions on the ground floor level occur together, gender-balanced street life will occur in the Spangen neighbourhood in Rotterdam too.

\subsubsection{Space and Cultures}

Urban design is often more concerned with appearance and aesthetics than with the interconnected logic of public, semi-public, and private spaces. It is primarily driven by a project's intention, cultural preferences, and ideologies. Little attention is paid to the new housing area's social function after it has been built, and the focus is simply on what sells and on short-term profits. Herein, the use of space syntax for urban design and planning can contribute to an understanding of how different types of people behave in new forms of urban areas (Major et al. 1999). Sometimes a project's intentions and ideologies do not correspond with its users' behaviours.

Why do many modernistic urban areas tend to be either gated communities or social-problem neighbourhoods? Segregated modernistic urban areas with high-income residents tend to be surrounded by fences, camera surveillance, or guards for protecting their properties. Presumably, most large modernistic housing estates tend to be designed by trans-spatial people, but they are populated mostly by spatially dependent people.

Spatially dependent people are the unemployed, the newly arrived immigrant, the housewife, the retired, the child, and the adolescent. They are dependent on what the vicinity offers because they spend a lot of time in and around their homes. Conversely, the trans-spatial people are students and working people. Often trans-spatial people have a social network that is not particularly place-bound or located in the vicinity of their homes, such as associations, clubs, organisations, etc. In their leisure time, trans-spatial people tend to participate in their activities within a much larger metric range than spatially dependent people (Rueb and Van Nes 2009). Therefore, the vicinity of trans-spatial people's own neighbourhood is not as important as it is for spatially dependent people. The spatial arrangement of evolved towns and cities seems to offer a complex set of sufficient spatial conditions for spatially dependent people, whereas the spatial arrangement in many post-War, modernistic high-rise neighbourhoods seems to be suitable for trans-spatial people. However, in most European countries, these post-War, high-rise flats are populated by spatially dependent people.

In general, the rationale behind how and where different types of people choose to live varies between cultures and nationalities. When investigating these aspects, a complex set of sufficient conditions is needed to understand a particular context. However, research on space and cultures is heavily context dependent.

Hermeneutic tradition: the conjunction of phenomena

Maybe $p, r$, or $s$ alone is sufficient for $q$ to occur. However, if $p, r$, and $s$ occur together, $q$ is certain to occur too.

As an example, we can apply the hermeneutic explanatory model to the case study of Jewish settlements in Manchester and Leeds in the nineteenth century, studied by Laura Vaughan and Alan Penn in 2001. The authors investigated the spatial features of the Jewish immigrants' settlement patterns when arriving in their host countries. As Vaughan and Penn found, the immigrants tended to locate themselves at the edge of economically active areas. This made it possible for the immigrants to participate in the economy of the host country and to slowly integrate into the local culture. Because Vaughan and Penn's study focused on urban areas in the nineteenth century, it provides knowledge about immigrants' behaviour and the distribution of poverty in evolved urban areas. As Vaughan and Penn's study shows, the socially privileged lived along the locally integrated main streets, whereas the lower classes were located 1 or 2 topological steps away from these streets. Immigrants also settled themselves 1-3 topological steps away from these main streets in order to have a chance for getting a job from the socially privileged (Vaughan and Penn 2001).

Hermeneutic explanatory model: The Manchester and the Leeds cases.

Maybe a neighbourhood with an integrated street network, economically active urban areas, or provision of cheap dwellings 1-3 topological steps away from integrated main streets was sufficient for Jewish immigrants to settle in Leeds and Manchester. However, if there was a neighbourhood in Leeds and Manchester in which these conditions occurred together, Jewish immigrants were certain to settle themselves in these areas. 


\subsubsection{Space and Political and Ethnic Conflicts}

In studies on political and ethnic conflicts, space syntax can only identify and describe the spatial features of where these types of conflicts take place. For example, in Belfast, the intentions to reduce the religious conflicts between Protestants and Catholics contributed to the construction of the peace lines. Research of this kind requires an understanding of the historic background of a certain culture and the political context as to why these walls were constructed. Space syntax can only describe the spatial features of the situation before and after the construction of the peace lines.

In contrast, for the Berlin Wall case, there were no cultural clashes. Here space syntax can be used to describe the spatial features on the location of various functions related to each political ideology. During the physical division of Berlin between 1961 and 1989, housing and political institutions were constructed along the spatially integrated streets in East Berlin. After the reunion of Berlin in 1989, the ground floor functions within these buildings were transformed back to shops. In a state-controlled economy, the incentive for shop owners to maximise their profits was lacking. Thus, the economic activity component of the theory of the natural movement economic process was distorted. Whereas in a free market economy, the marketplace generates capitalistic activities, in the communist ideology of Karl Marx, it was common practice to locate residences and political institutions along the most integrated streets in city and town centres. This Marxist ideology was used in the rebuilding and regenerating of Eastern Block cities behind the Iron Curtain (Van Nes 2002, p. 276).

Whether or not the spatial setup in built environments can provoke political activities is difficult to comment on, and systematic research on this issue is lacking. However, political forces and organisational constraints can overpower spatial forces in built environments. In a state-controlled economy, there is almost no incentive for profit maximising. According to the ideology of former communist countries, a market square or city centre was perceived as a generator of "capitalistic activities" (Lefebvre 1991, p. 101ff), and therefore, large housing estates were often built in their historic city centres to reduce the possibilities for these so-called market activities. The former East German centres of Alexanderplatz in Berlin and the Dessau city centre provide examples of how a political system of this kind can overpower the generative power of the natural movement economic process. In the same way, a strong planning system on different levels-national, regional, or municipal levels - as well as organisational constraints can block the natural choice to locate economic activities at strategically optimal locations. Below we use Dessau centre during the Deutsche Demokratische Republik (DDR) period as a context-dependent case to show an explanatory model related to the effects of a political system.

Hermeneutic explanatory model: the conjunction of phenomena

$<$ Maybe $p, r$, or $s$ alone is sufficient for $q$ to occur. However, if $p, r$, and $s$ occur together, $q$ is certain to occur too.> Hermeneutic explanatory model: The Dessau centre case during the DDR period from 1945 to 1989.

Maybe the intention to obstruct capitalistic activities, the intention to solve the dwelling shortages, or the intention to rebuild the destroyed city centre alone was sufficient for there being no shopping activities in Dessau centre between 1945 and 1989. However, if the intention to obstruct capitalistic activities, the intention to solve the dwelling shortages, and the intention to rebuild the destroyed city centre occurred together, there was certain to be no shopping activities in Dessau centre between 1945 and 1989.

In a research project on how diverging ideologies impact the location of functions in relation to spatial integration, four different settlements were analysed in the Norwegian archipelago of Svalbard. These northernmost permanently inhabited settlements in the Arctic region are located $1,300 \mathrm{~km}$ from the North Pole. Two of these are Norwegian settlements. Longyearbyen and $\mathrm{Ny}$-Ålesund were founded as coal-mining towns, and today they are international polar research stations. The other two, Pyramiden and Barentsburg, are Russian coal-mining towns constructed during the former Soviet Union. All four towns are separated from each other due to the extreme climate and landscapes. Therefore, they represent unique cases for understanding the relationship between economic systems, two diverging political ideologies, and built form and function. Each town has its own local community with limited possibilities to interact with the other towns.

The space syntax analysis reveals that political ideology influences the types and distribution patterns of public functions. Figure 6.16 shows a space syntax analysis of Longyearbyen and Pyramiden. In capitalistic, liberated economy types of settlements, public functions along well-integrated streets consist of commercial facilities such as shops, bars, restaurants, Vinmonopolet (Norwegian state monopolised alcohol sales shops), offices, and educational, governmental, and cultural institutions. Because commercial functions are lacking in (former) soviet/communist settlements, housing, cultural, and social facilities, as well as Lenin's statue are located along the most integrated streets in communistic, state-controlled economy types of settlements. These results show that space syntax is used here to describe and identify the spatial features of space use based on different ideologies and cultures. Research of this kind is heavily context-dependent and requires an understanding of the political ideology at issue (De Koning and Van Nes 2019a, b). 
Fig. 6.16 Global integration analyses of the towns of Longyearbyen and Pyramiden showing the locations of functions (De Koning and Van Nes 2019a, b) (a) Longyearbyen

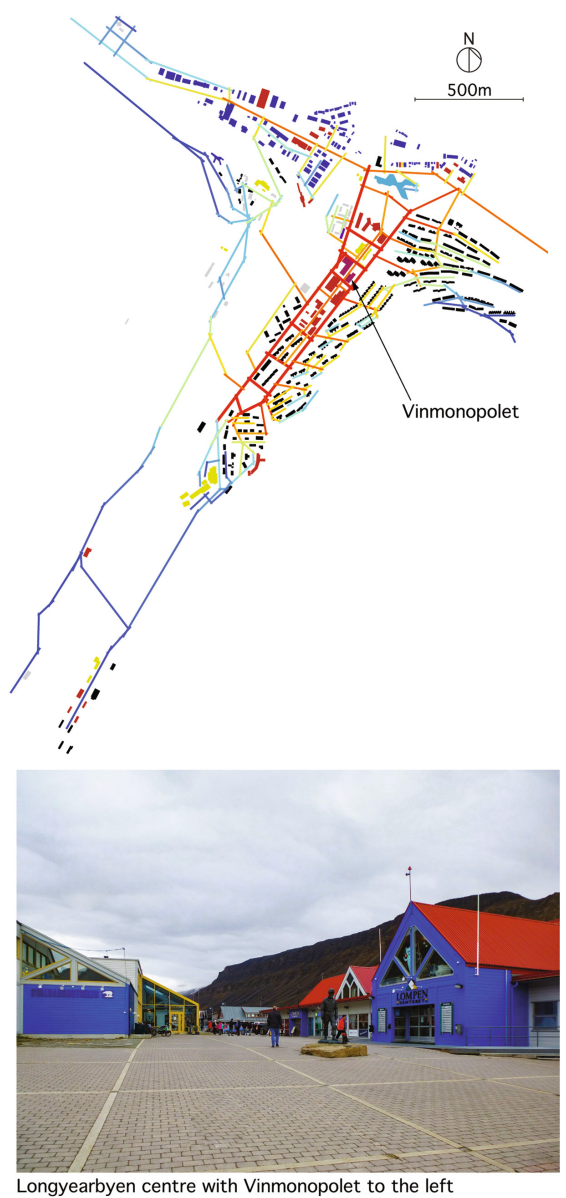

(b) Pyramiden
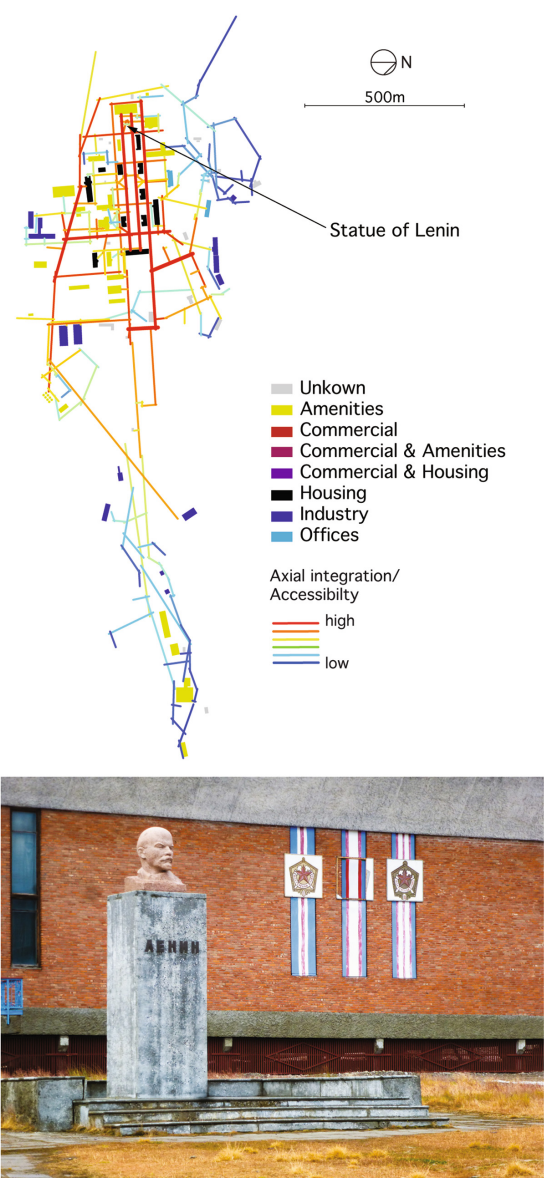

tatue of Lenin in front of the Community House

For Longyearbyen, the street with the highest spatial integration is a sufficient condition for the location of the supermarket with the alcohol sales point Vinmonopolet. For Pyramiden, the intention to make the Lenin statue visible to the inhabitants was a necessary condition for the location of the Lenin statue at the highest integrated street.

Understanding the location pattern of activities in the centre of Pyramiden requires a hermeneutic approach, whereas the explanation for the commercial activities in Longyearbyen requires a positivistic approach.

Hermeneutic explanatory model: the necessary condition

$p$ is a necessary condition for $q$.

Hermeneutic explanatory model: The explanatory model for the location of the Lenin statue in Pyramiden

The intention to be a showcase for the ideal communistic settlement is a necessary condition for the location of the Lenin statue in Pyramiden's most integrated space.

For Longyearbyen, the street with the highest integration is a sufficient condition for the location of the supermarket with the alcohol sales point. This is in line with the theory of the natural movement economic process.

Positivist explanatory model: the sufficient condition

$p$ is a sufficient condition for $q$.

Positivist explanatory model: The Longyearbyen case.

The street with the highest spatial integration of the street network is a sufficient condition for the location of the supermarket with the alcohol sales point in Longyearbyen.

In a study about ethnic conflicts, it was shown how a cognitive boundary with no physical presence has affected life in the cities of Jaffa and Tel Aviv for many years. The cognitive border existed not only during its time of existence (1921-1950), but for many decades after it was erased from all official documents. In 1921, the national aspirations of Jews in Jaffa, embraced by the local British Mandate government, triggered a segregation process that resulted in an official administrative split of Jaffa's urban area. In this way, the 'Hebrew' city of Tel Aviv on Jaffa's northern parts was created. This administrative division had a clear ethnic character. The entire city was divided into clearly defined 'Jewish' and 'Arab' geographical entities, and this influenced the development of the two municipalities as well as the daily lives of their populations. 
Figure 6.17 shows a normalised segment integration (NAIN) of Jaffa and Tel Aviv from 1944 with the paper border (left) and without the paper border (right) (Aleksandrowicz et al. 2018). After the $1948 \mathrm{War}$ in Palestine, which led to the flight of almost all of Jaffa's Arab population and the annexation of the Jaffa area to Tel Aviv, the united city continued to resemble a divided city. The former areas of Jaffa remained relatively underdeveloped and neglected for decades (Aleksandrowicz et.al 2018). By combining spatial analysis and historical research, this study revealed how the 'paper boundary' that was drawn between Jaffa and Tel Aviv, in 1921, transformed the life of Arabs and Jews in the two cities in a way that undermined the physical unity of the urban fabric and the spatial potential of its street network.

Fig. 6.17 The division of Jaffa and Tel Aviv, in 1944-(left) NAIN analysis of Jaffa and Tel Aviv, computed including the 'paper boundary'; (right) NAIN analysis of Jaffa and Tel Aviv, where the unmanipulated axial map was computed (Aleksandrowicz et al. 2018)

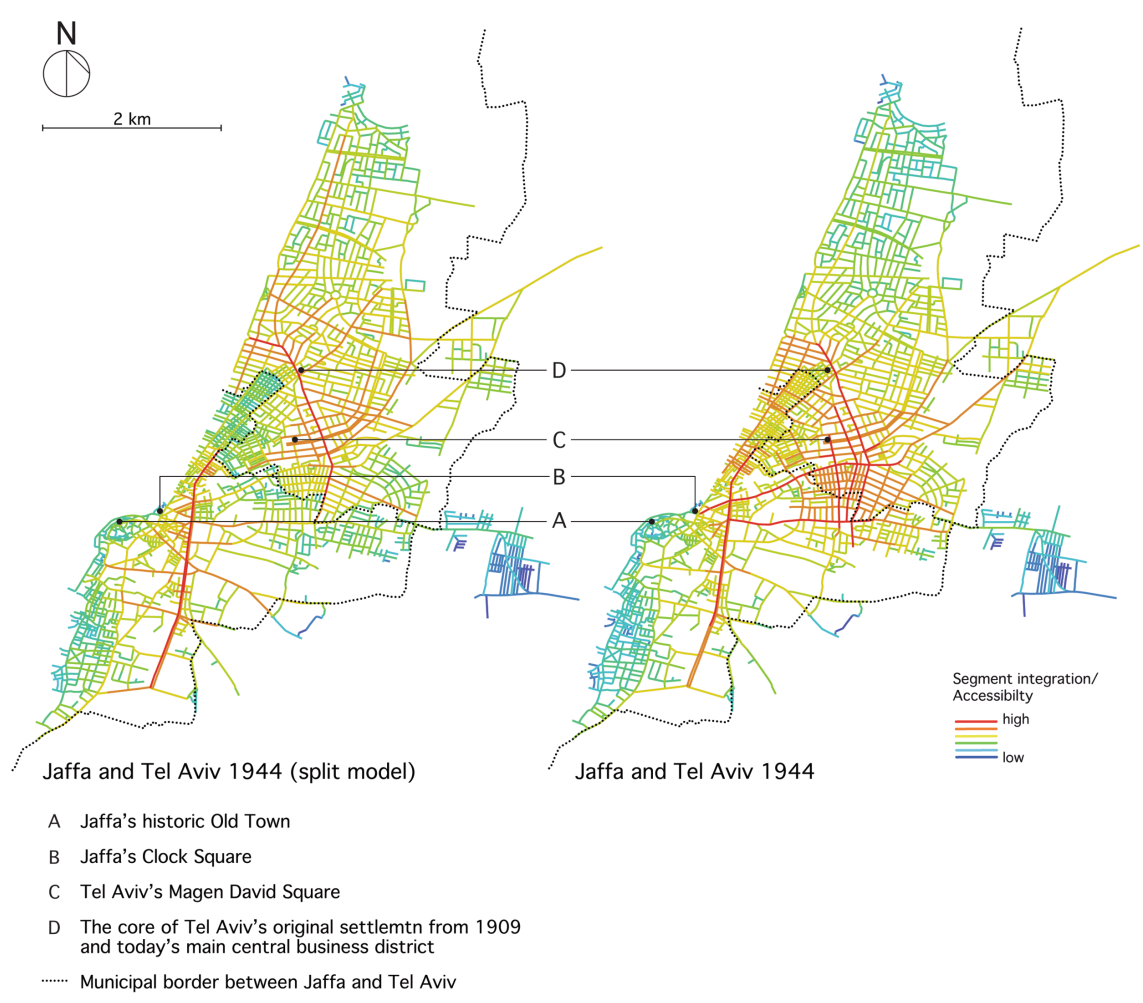

The creation of the municipal border led to the cognitive marginalisation of the spatially centrally located Manshiya neighbourhood, and later to its deterioration and eventual destruction. Ironically, the destruction of Manshiya gave a belated physical expression to the historic cognitive separation between the centres of Jaffa and Tel Aviv, working against the wish to unite the two cities into a single urban entity after 1948 (Aleksandrowicz et al. 2018).

Hermeneutic explanatory model: the conjunction of phenomena

Maybe $p, r$, or $s$ alone is sufficient for $q$ to occur. However, if $p, r$, and $s$ occur together, $q$ is certain to occur too.

Hermeneutic explanatory model: The Jaffa-Tel Aviv case.

Maybe the intention to create the Hebrew city, the intention to make an ethnic division between Jews and Arabs, or the intention to make an administrative division alone was sufficient for the paper borders to be drawn in 1921. However, when the intention to create the Hebrew city, the intention to make an ethnic division between Jews and Arabs, and the intention to make an administrative division occurred together, the paper borders from 1921 were sure to be drawn too.

Research on space and political or religious issues requires an understanding of the underlying ideologies for each particular context. Everything outside market rationality, namely the necessary conditions for where a particular culture or political ideology places their main symbols, needs to be identified. The same is true for the complex set of sufficient conditions.

\subsection{Context-Dependence: How Space Syntax Theories Can Be Distorted}

Space syntax offers specific concepts on urban space that can be used when analysing built environments' spatial changes over time independent of political and cultural contexts. Therefore, space syntax is able to describe the spatial properties concerning where certain activities take place within specific political or cultural contexts. 
Shops do not always locate themselves along the highest integrated street, and therefore, the local planning context needs to be investigated in these cases. Likewise, the flow of human movement does not always correspond with the degree of spatial integration. This yields a representation of the local cultural or political context. Figure 6.18 illustrates how the theory of the natural movement economic process can be distorted by political and organisational constraints or by ethnic conflicts. The Netherlands, for example, has a strong planning system that works well at the national level, provincial level, and local level. It is, for example, difficult to locate or to invest in highly integrated areas when they are affected by national protection plans or conflicting municipal interests (Bruyns et al. 2007, p. 86).

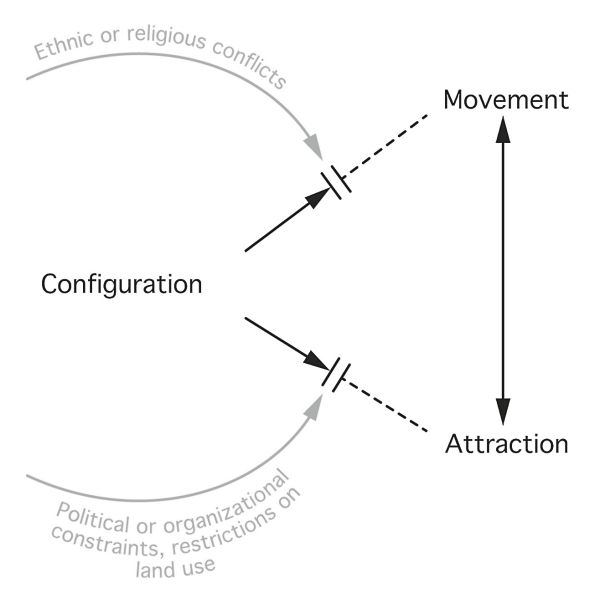

Fig. 6.18 Interferences in the theory of the natural movement economic process

In contrast, Norway has a weak planning system on all levels. Therefore, urban development is steered by market forces and the municipality functions as a facilitator and mediator for the development of industrial areas, new housing estates, and shopping malls (Falleth et al. 1995). Thus, a country's planning system or political forces can influence the location pattern of economic-related activities. These aspects have to be taken into account in research as well as in planning of urban areas.

Highly integrated urban areas do not always have a high degree of building density or a high degree of land use diversity. Likewise, high building density does not always entail multi-functional land use. Figure 6.19 shows the distortions in the theory of the natural urban transformation process. Gaining understandings of all of these distortions depends on each local or national context. There might be rigid restrictions on the local planning regulations, restrictions from conservation authorities, or laws and rules that block the desired intentions in the planning process.

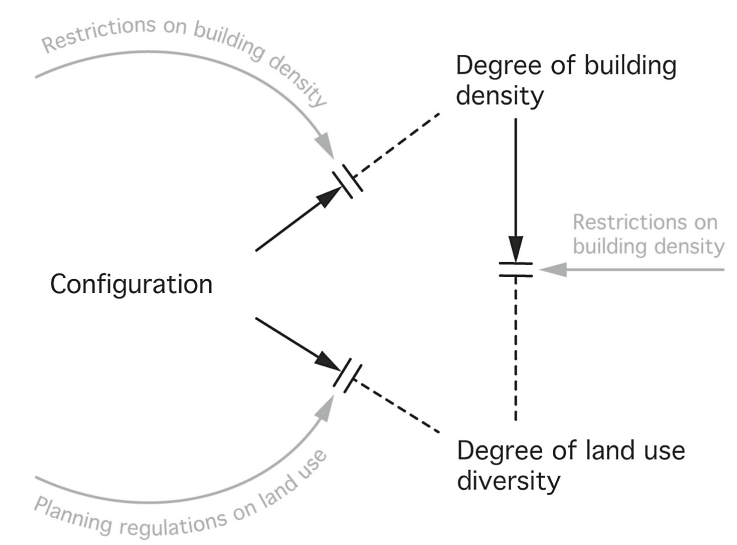

Fig. 6.19 Interferences in the theory of the natural urban transformation process

Research on these issues requires an understanding of a country's planning systems and its associated laws and policies. Likewise, understandings of a particular culture and tradition can provide explanations on various interferences. Extreme natural conditions (flooding or land-slide risks) or religion (practices and rituals) also contribute to regulations that might distort the relationship between integration, movement, and the location of attractors. It is all about identifying the necessary condition or complex set of sufficient conditions for each local context. 


\subsection{A Significant Piece Towards a Comprehensive Theory on the Built Environment}

What does space syntax add to studies on the built environment? At the very least, space syntax offers concise spatial tools to measure spatial changes in built environments independent of context-related situations where cultural aspects must be taken into account (Yamu et al. 2021). Therefore, it is able to find some spatial evidence for various presumptions and observations.

What can space syntax not grasp in studies on the built environment? Even though space syntax offers precise spatial concepts for one to operate with, it cannot analyse the place character, the sphere, the ambience, or the symbolic meaning of the built form. Likewise, explanations and understandings as to why a settlement was established at a certain natural location cannot be offered using space syntax analyses. However, space syntax can analyse the configurative structure of their spatial set-up as an independent factor of the built form's symbolic meaning. Therefore, space syntax deals with place structure and not with place character. Analyses of place character require a genuine understanding and insight into a society's cultural background and spiritual traditions in the present as well as in the past.

According to David Seamon, understanding the built environment as an integral structure of human life must consider the following three dimensions: geographical ensemble (the physical environment), people in place (human actions, meanings, and intentions), and genius loci (the spirit of the place) (Seamon 2011). The space syntax method is able to analyse the spatial structure of the geographical ensemble and correlate these results with registrations of certain kinds of activities by people in place. These activities relate mostly to human actions and certain kinds of human perceptions. However, the symbolic meaning of urban form and a place's genius loci cannot be grasped by space syntax. According to Seamon, the added value from space syntax is that the method can demonstrate that the spatial arrangement of the built environment can describe lively and well-used public spaces or empty and lifeless public spaces. The experience of these issues relates to the phenomenological experience of a place (Seamon 2020, p. 7).

While researchers taking a phenomenological or architectural extensionalistic approach seek to describe the underlying essential qualities of human experience and the world where these experiences occur (Seamon 1994, p. 37 and Seamon 2012), researchers with a space syntax approach seek to identify the spatial conditions for lively or quiet urban squares, streets, neighbourhoods, etc. Understanding the spatial conditions of pedestrian flow rates and degree of urban vitality is also an essential component for understanding the sphere of a place. This aspect is lacking in much of the literature on place phenomenology. When developing a systematic and comprehensive architecture theory where all kinds of aspects concerning how human beings exist in their life world are taken into account, space syntax is one essential part in providing an understanding of the life of places. Even though Norberg-Schulz criticises taking a quantitative approach in studies on the built environment (Norberg-Schulz 1967, p. 202), a space syntax approach can at least provide some exact evidence on how some spatial components of the built environment create lively or quiet places. Therefore, generalisations and theory building on built environments requires multiple research approaches incorporating conflicting epistemological and ontological assumptions (Seamon 2011).

Are there intentions in the way the built environment is constructed? Or are there intentions behind how human beings behave in the built environment? Intentions seem to play a role in constructing the elements shaping a place's character. These elements are defined to be the architectural style of buildings and their ornaments such as the shape and forms of windows, doors, plinths, roofs, and walls. Other artefacts such as the texture of the streets, windows, fountains, etc. also shape a place's character. However, studies concerning place structure tend to focus on the observed human behaviour rather than on human intentions and spiritual traditions. The intentions can be found in the kind of rationality people have in the way they behave in urban space and how they organise the spatial set-up for their daily activities. As Hillier and Hanson write, a space syntax approach is about understanding "the social content of spatial patterning and the spatial content of social patterning" (Hillier and Hanson 1984, pp. x-xi). This is a small, but significant contribution towards a comprehensive theory on built environments. 


\subsection{Epilogue: A Thought Experiment for the Sustainable City Debate}

How can the theoretical aspects of space syntax be applied in the sustainable city debate? In this epilogue section, we carry out a thought experiment on how spatial parameters can encourage or limit sustainable urban transformation and sustainable mobility means. We further connect the sustainable city debate to existing space syntax theories and understandings on the relationship between space and society.

The challenge is that sustainable development concerns both normative and descriptive issues, and here we are dealing with present as well as future needs. In order to predict future needs, a descriptive approach is needed from a present context. We have to explain or understand what a sustainable city is as an 'object' and how this 'object' can generate sustainable ways of behaviour and sustainable urbanisation processes. Sustainable urbanisation processes can include low energy use for transport, high walkability potentials ('last mile connectivity'), low consumption of nature resources, low degree of urban sprawl into the countryside, safety, possibilities for social contact, healthy cities, and a natural mixture of all types of people in public spaces.

To date, precision in the spatial elements for describing a sustainable city is missing, and this lack of precision of the spatial elements leads to a lack of spatial analysis methods, which in turn, leads to a lack of evidence on the relationship between the built form and the sustainable behaviour of its users. This again leads to weak explanations on what a sustainable city is, even though such explanations are needed to make operational policies and to develop plans for enhancing sustainable urbanisation processes. However, some attempts for describing urban sustainability were made at the end of the 1990s. The compact city model is proposed to encourage sustainable ways of living (Roger 1999; Jenks et al. 1996). However, precise definitions of the compact city's spatial components are missing, and mostly normative statements on how to plan and build a sustainable city have been proposed. Understanding how spatial forces can generate sustainable urban processes is lacking in most writings on urban sustainability connected to the debate on urban compactness.

Urban compactness can be approached from a space syntax point of view because compactness is a topological property. In a very loose manner of speaking, the word compact describes that which is closely and firmly united, pressed together, dense, fine-grained, and packed into a small space (Encyclopaedia Britannica 1955). The answers might result in an understanding of how the spatial configuration of an urban street network generates movement and visibility and how it influences the dispersal of economic and social activities.

Most writings on urban sustainability and compact cities describe the problem cities are facing today with regards to sustainability (Roger 1999; Jenks et al. 1996; Calthorpe 1993). Most authors offer quick normative proposals as to how one should design a compact city. However, what is missing in these writings is a concise understanding of how compact cities function with regards to social and economic activities and to the power of the urban street network itself for generating human movement by foot or vehicle and the subsequent social and economic effects of this.

According to the Brundtland report, one of the basic environmental problems of modern cities is high energy use for transportation - generally speaking, private car dependency (Brundtland Commission 1987). Private car dependency not only relates to a lack of available and efficient public transport, but also to the spatial structure of the street and road network. This spatial structure influences the way people move, either by foot, public transport, bicycle, or by car, and how urban functions are dispersed. Building density and a mixture of activities first and foremost depend on the spatial structure of an urban street network. The relationship between the density of the built mass and transportation is another key to achieving a sustainable city (Rådberg 1996; Næss 2006a, b; Saglie 1998), and the higher the density of the built mass, the greater the mix of various urban functions and the greater the energy savings for transport per person (Roger 1999; Næss 2006b).

However, how a street and road network's structure can generate private car dependency is seldom taken into account in current research and planning practice.

Social interaction and physical movement certainly shape a built environment, and there are interdependencies between the physical built environment and both economically and socially motivated movement. Thus, physical form and social activity influence each other. Urban compactness thus should be understood in terms of movement and interaction and greater inter-accessibility of urban functions within a short metric distance.

An account of compactness and sustainability in configurative terms has to be descriptive because it concerns both structural and social aspects. While Alexander (1965) concentrated on structural aspects and Jacobs (1960) accounted for social aspects, a space syntax approach offers mathematical means to consider the two aspects at the same time. Compactness is thus understood in terms of space. 


\subsubsection{Describing Compactness with Space Syntax}

The following examples will demonstrate how a compact vital city can be described in a concise and sustainable manner. In his book Towards an Urban Renaissance, Richard Rogers illustrates some principles on compact urban centres (Roger 1999, p. 53). Compact urban centres are indicated as grey circles concentrated around a public transport hub. The grey colour indicates high urban compactness, probably in terms of high density of the built mass. The finer spatial content inside these circles is missing, and Rogers's illustration does not show how these centres can function socially and economically in a sustainable manner. Likewise, a concise understanding of how each centre relates to its surrounding areas and the whole city is lacking. In general, the fine-grained street grid and its interconnectivity are not taken into consideration at all.

Figure 6.20a shows how compact urban centres are described in most planning and policy documents. Often strategic development plans for densification consist of colouring the appointed areas in a darker colour than the rest of the city. In most cases, these areas tend to surround metro or train stations. The aim is to show the areas where the density of the buildings can be increased. However, fine-grained urban data on how the local street network is connected to the public transport hub are often lacking.

Fig. 6.20 A way of visualising urban compactness in most planning documents (a) and a space syntax description of urban compactness in terms of degrees of spatial integration in the pedestrian-based shopping areas in Oslo (b)

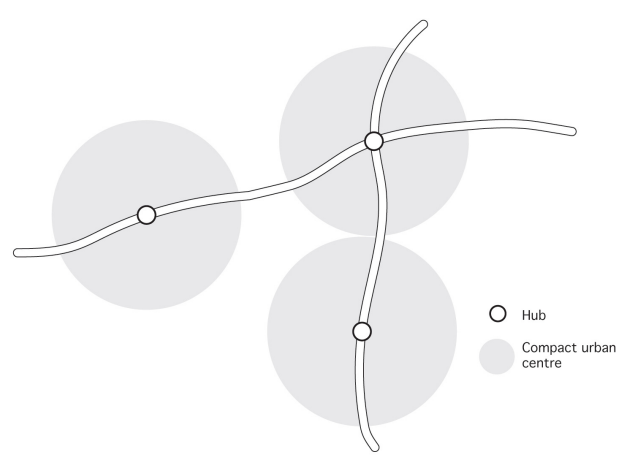

(a) Visualization of urban compactness in most planning documents

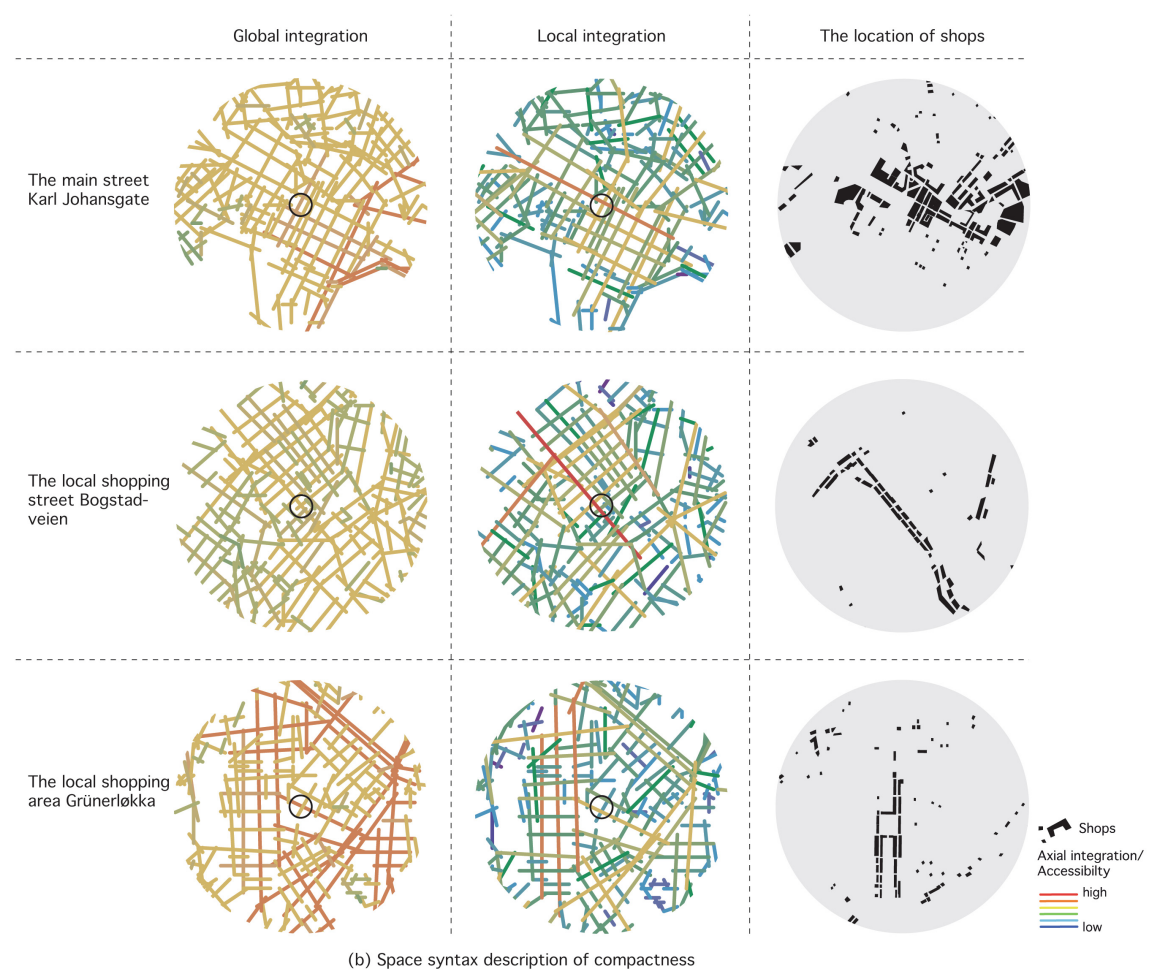


Below in Fig. 6.20, local and global integration analyses are shown within a radius of $1 \mathrm{~km}$ of the street networks of the three main pedestrian-based shopping areas in Oslo. The highest local integration is on the main shopping streets. The location patterns of the areas' shops are shown at the right side of the figure. The black ovals indicate large shopping centres. All of these areas have in common that they provide a dense and well-connected street network within a short metric distance of $1 \mathrm{~km}$. This is an indication of compact sustainable urban areas. The density of the street network is high and the local and global integration values are also high.

Figure 6.21 depicts two car-based shopping centres located at the globally highly integrated Ring road 3 in Oslo. The local integration values and the density of the street network are low in these two areas. The street network has a tree structure, and the building density is low. This does not encourage movement by foot. Urban centres with this kind of spatial structure are examples of unsustainable urban areas due to their low degree of walkability. In comparison with the grey circles used in most planning documents (Fig. 6.20 top), a space syntax approach (Figs. 6.20 bottom and 6.21) shows both the fine-grained detail of the street and road network and their degree of inter-accessibility on a local and city-wide scale.

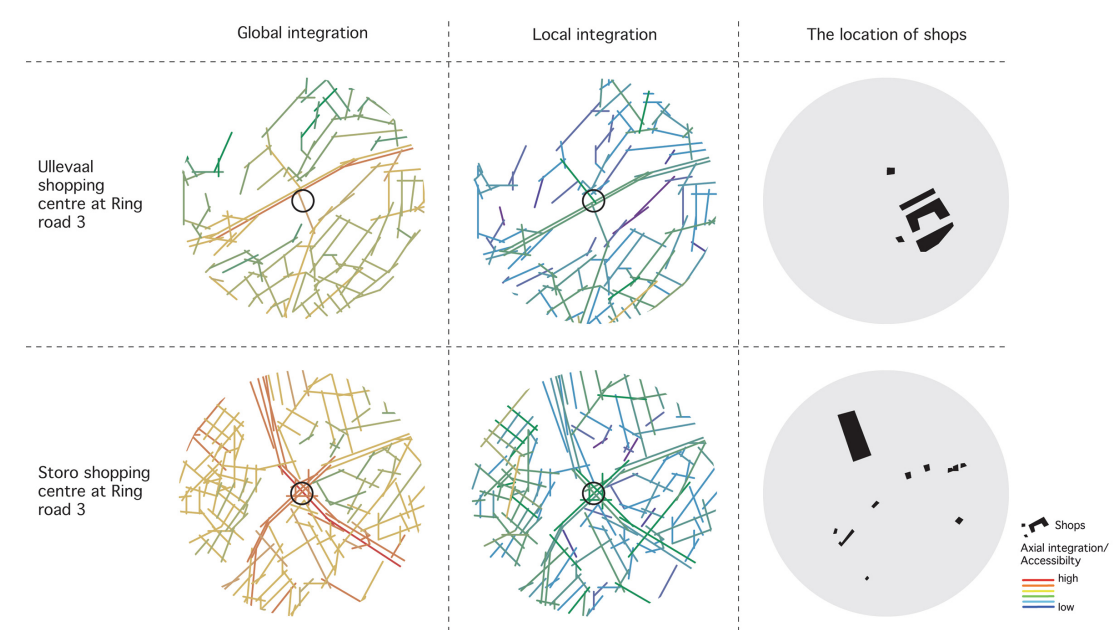

Fig. 6.21 A space syntax description of urban compactness in terms of degrees of spatial integration in two car-based shopping centres in Oslo

To illustrate the differences, Fig. 6.22 shows figure-ground diagram of Bogstadveien as an example of a pedestrian-based shopping street and Storo Senteret as an example of a car-based shopping centre in Oslo. The street network density, building density, and land use diversity are high in the Bogstadveien area, whereas they are low in the Storo Senteret area. All buildings have entrances and windows facing towards the streets in Bogstadveien. Conversely, most of the areas around Storo Senteret are covered by asphalt and are car dominated. The area is known to be pedestrian unfriendly, and the large shopping centre is turned inward. Storo Senteret has few entrances, and they are hard to find for pedestrians. For the car drivers, access to the shopping centre is direct from the parking garage.

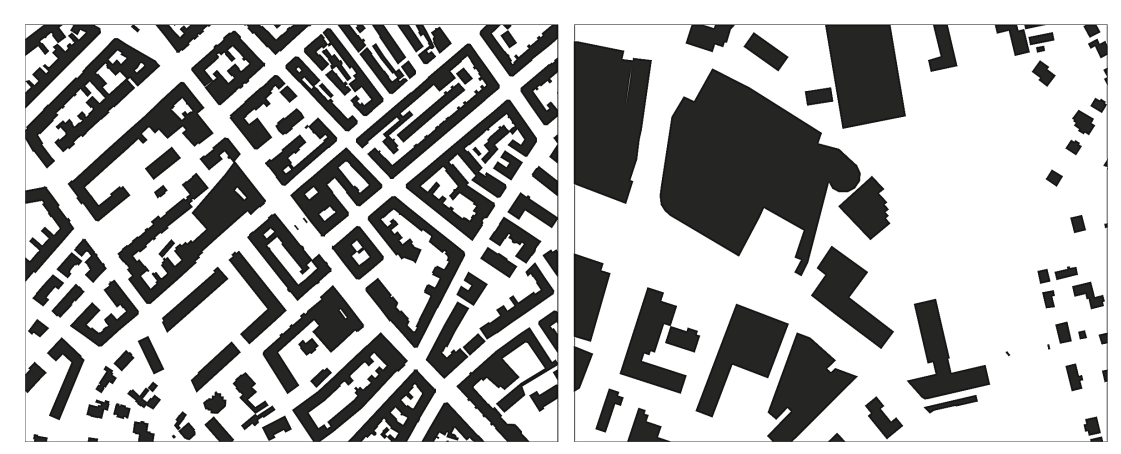

Fig. 6.22 Left: Bogstadveien as an example of a pedestrian-based shopping street. Right: Storo Senteret as an example of a car-based shopping centre 
Furthermore, in recent research with the case studies of Zürich in Switzerland and Bergen in Norway, correlations were found between the street network configuration and energy use for transport. Neighbourhoods with high values for the angular choice analysis and with a low and high metric radius have low values on energy use for transport. These kinds of spatial configurations enhance walking, cycling, and the use of public transport. The highly integrated main routes are suitable for implementing public transport means such as trams or buses. Conversely, neighbourhoods with low values on angular choice with a low metric radius enhance private car dependency. Likewise, the same accounts when the neighbourhood has high values on the angular choice values but low values on the angular choice with a high metric radius (De Koning and Van Nes 2019a, b).

Hence, criteria for successful compact pedestrian-based urban centres imply high density of the street network within a short metric distance and interconnectivity between local streets, main routes, and the road network of the whole city. The spatial structure of the mobility network can thus encourage or block sustainable modes of transportation. Therefore, large interventions on the mobility network can affect the socio-economic life in cities.

In traffic planning, there is, however, a contradiction between traffic safety and social safety. Banning cars from urban centres contributes to 'dead' town centres, in particular after shops are closed. On the contrary, having high car-accessibility to urban centres contributes to traffic accidents and high levels of noise and pollution. Seemingly, the key is to 'humanise' the private car in planning practice with the purpose of retaining lively streets and economically vital town centres. Otherwise, overly intense restrictions on private car use in town centres can contribute to the development of out-of-town shopping centres along the highways.

In Copenhagen, the influence of Jan Gehl's research has contributed to a new shift in planning. The concept 'To Copenhagenize' consists of reducing the role of the accessibility of private cars and enhancing walking and cycling. The role of the road engineer is reduced, and the focus is on creating urban spaces as places to stay and perform social activities rather than just spaces to travel through (Gehl 2010, p. 13).

In this context, the construction of new road links can have a comprehensive impact on a city's spatial structure and socio-economic activities. Whether or not a new road enhances sustainable means of mobility depends on at least two factors - the kind of street network the new road is imposed upon and how this imposition comes about. Therefore, the methods applied here and the results thus obtained can be useful for predicting certain consequences of new road projects.

So far, rigid traffic safety standards seem to steer how new motorways or main routes through urban areas are interconnected to their local vicinity. In addition to this, research using space syntax can shed some light on the role of the road engineer in urban development. The road engineers create the necessary spatial framework for the socio-economic life in built environments. It all depends on how their planned new road links segregate, integrate, connect, or disconnect the urban areas they are imposed upon. Thus, everything depends on various degrees of connectivity, adjacency, visibility, and accessibility (Van Nes 2021). In addition, the effects of the relevant kinds of changes can be explained in terms of changes in the location patterns of shops and retail outlets as well as the various means of mobility-whether they are sustainable or not. In this case, the theory of the natural movement economic process with the spatial configuration of the street network as a sufficient condition is relevant for explaining the impact of the spatial changes of the street network on built environments.

\subsubsection{The Street-Building Interface and Its Impact on Street Life and Safety}

During the last two decades, living in central urban areas has become popular in Europe, and this 'urban renaissance' has contributed to an increase in estate and property prices in inner city areas. Many people are seeking urban areas with high social, cultural, and spatial diversity; short distances from dwellings to work, leisure, and cultural activities; and the ability to take advantage of all the opportunities a city has to offer (Roger 1999). In addition, these trends are in line with new planning policies where the aim is to densify existing built-up areas instead of increasing urban sprawl into the countryside. The idea is that high diversity and density of various kinds of land use, functions, and cultural activities will contribute to vital and lively urban areas and economic growth (Roger 1999, p. 45). However, the topological spatial relationship between private and public space and its impact on urban street life is a forgotten aspect in most contemporary writings on compact cities, urban design, and urban planning.

Architects tend to emphasise the feeling of privacy within the context of modern living in their design projects. Examples of the kinds of built environments these tendencies generate can be seen in Fig. 6.23. Often an explanation can be found in the individualisation process of human beings over the last 60 years in western society. There is a belief that there is a need for a high degree of privacy when a large group of people are living together in a small number of square metres. The effect is that dwelling entrances and windows are hidden away from public streets. 

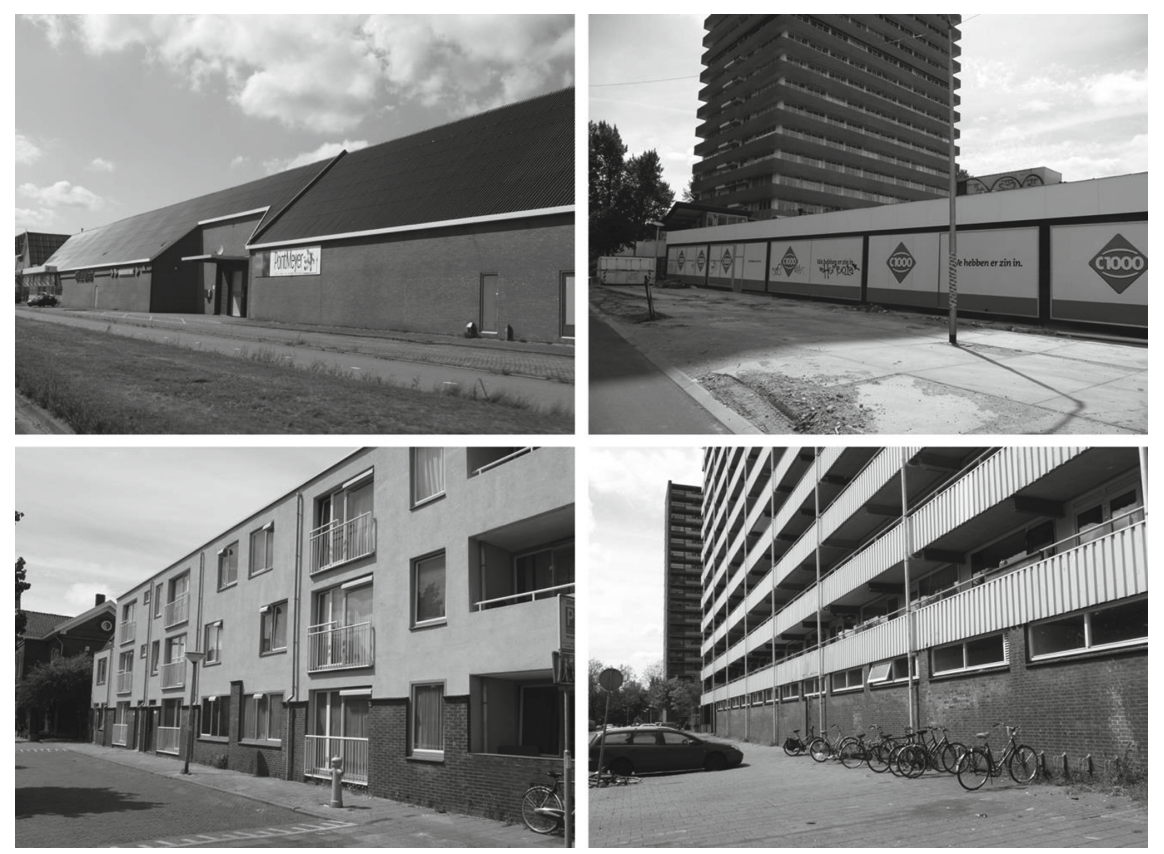

Fig. 6.23 Modern building facades turned away from streets

Micro-spatial relationships on the street plinth level play a significant role in the socio-economic life of human beings. The architecture of building openings shapes the spatial possibilities or limitations for visibility, accessibility, and permeability and thus influences the various degrees of contact possibilities between the private and public spheres. In particular, urban renewal projects, modern housing areas, and new large-scale urban development projects tend to lack adjacency, permeability, and intervisibility between buildings and streets. This has negative effects both on street life and the degree of perceived safety of these areas. Jacobs already advocated that buildings should have 'eyes on the streets' in the 1960s. In addition, streets with buildings lacking 'active frontage' reduce the attractiveness of walkability in neighbourhoods, which again enhances private car dependency and perceived unsafe streets. Figure 6.24 shows an example of a recently finished neighbourhood in Delft. The 'active frontages' are orientated towards the back gardens where the private life takes place, whereas the entrances on the homes' front-sides are turned away from the streets.
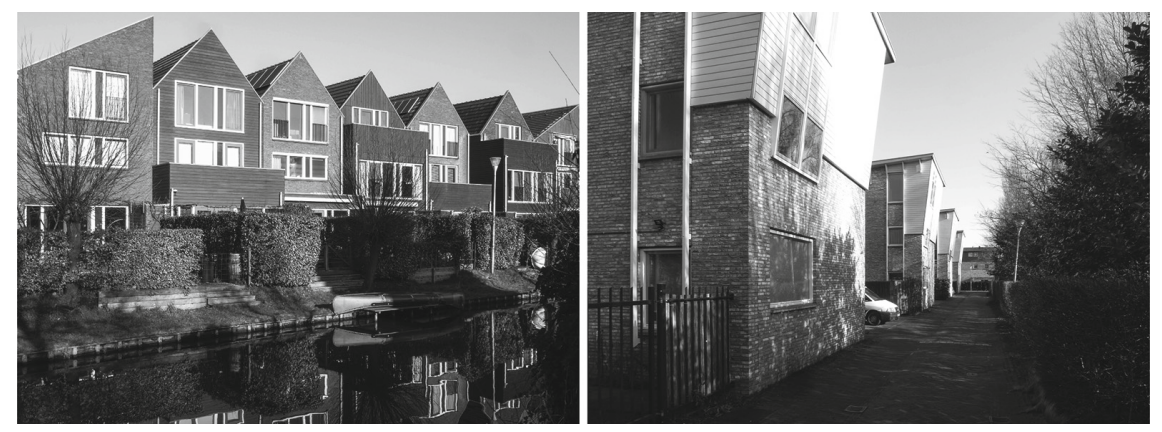

Fig. 6.24 Modern residential area in Delft. The dwelling and the private life are oriented towards the canals (left) on the house's backside, while all the streets are unconstituted on the home's front side (right)

High values on the FSI are not always a condition for safe and lively streets. In the 'vertical city' of Hong Kong, there are several examples of new housing projects that are not well connected to the street. Even though the number of apartments is high, there is little street life at the street plinth level (Hwang 2006). Stacking apartments often contributes to vertical sprawl, but degrees of street life seems to depend on how these flats' entrances are connected to the street. However, the degree of interconnectivity and the topologically shallow public-private interface is often forgotten. All of these activities depend on 
the spatial configuration on the plinth or built-up street sides. Figure 6.25 shows images of a recently finished housing project near Shanhanguan in China. The number of inhabitants in this neighbourhood is high, but the streets lack shops and places for contact.

Fig. 6.25 Images of a vertical neighbourhood in Shanhaiguan in China
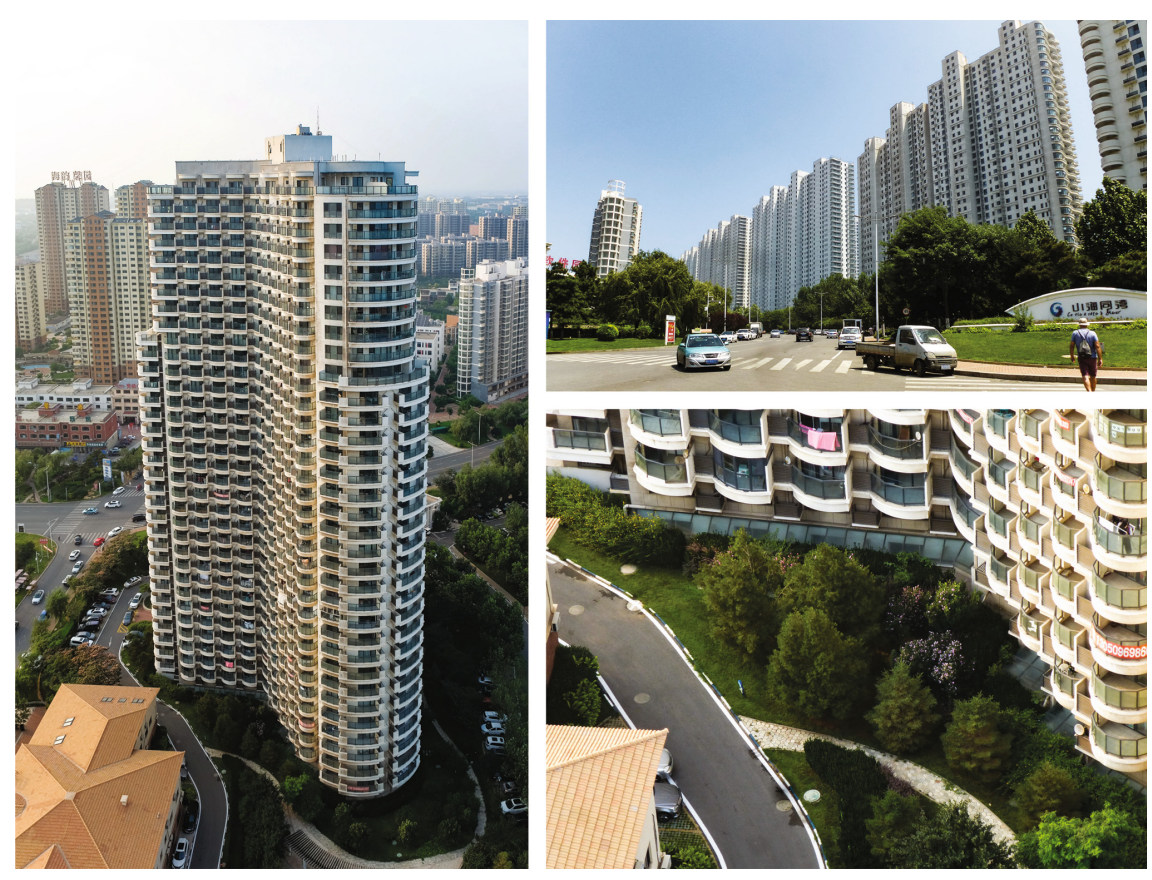

The micro-spatial conditions of the street segment are inter-related to the macro-spatial conditions of the city's street network. The definition and operationalisation of the micro-scale conditions is, however, still in a preliminary phase and is an area that can be improved upon in the near future. At the very least, some concepts useful in urbanism have been introduced and have brought significant aspects into the urban sustainability and compact city debate. This is not only important for urban research, but also for the design and planning of our cities.

The quality of the building-street interface in our built environments seems to be affected by a division of responsibilities. The road engineer is concerned only about the street and road dimensions, whereas the project developers are concerned with the maximum short-term profits they can get from their plots. The municipality lacks operational concepts and tools to ensure safe and vital streets in their policy and planning documents and in the way building permissions are given.

If we connect all of these above-mentioned issues to theory building and complex conditions (see Sect. 6.5), street life and the perception of safety appear to be dependent on a complex set of sufficient conditions. Perception of safety seems to depends on the presence of people in the streets. Here we use von Wright's conjunction of a phenomenon, and again we are dependent on a particular case. Here we use the results from a research project on the perception of the street safety of six different neighbourhoods in Bergen centre (Rønneberg Nordhov et al. 2019).

Hermeneutic explanatory model.

Maybe $p, r, s, t$, or $u$ alone is sufficient for $q$ to occur. However, if all of them occur together, $q$ is certain to occur too.

Hermeneutic explanatory model of the Bergen case

Maybe constituted streets, short topological depth between private and public space, highly intervisible streets, high density of entrances, or high street network integration on all scale levels alone is sufficient for the locals' perception of safe streets in Bergen. However, if all these spatial aspects occur together, the streets will be perceived as safe by the locals in Bergen. 


\subsubsection{Spatial Structure, Configuration, and Sustainability}

At least since the time of the Industrial Revolution, we have seen how comprehensive technical inventions have affected the spatial structure of built environments, and conversely how spatial products have affected social and economic behaviour. Humans are able to change their built environment, and they have purposes and intentions in doing so. It is not always clear what the intentions are, but those concerning economic activities strive for profit maximising. This makes it possible to predict some of the impacts on society based on the spatial structure of the built environment.

Alexander Cuthbert claims that writings in the urban design discipline should make a connection to political economy. As he writes, "The more recent form-spatial political economy offers promise in that its fundamental concern with the processes through which social space is produced, reproduced, transformed and exchanged, intersects neatly with how specific forms of social space arise" (Cuthbert 2007, p. 211). Aiming at the creation of urban areas that can develop in a sustainable way cannot ignore the behaviour of producers and consumers and the way the built environment influences them. From a space syntax point of view, understanding what an urban area's sustainable development consists of depends on an appropriate account of the geometrical and topological structure of its street network.

City growth can affect the global integration core and the development of local integration cores. A space syntax approach can help to understand why the location of the vital main centre changes and why from a spatial point of view the old centre becomes segregated. In many cases, large amounts of resources are used to revitalise segregated old historic urban centres. These activities consist of putting new functions in old buildings, restoring historic buildings, shining up the street-scape and squares, and making policies to adjust new buildings to existing ones. What is missing in most plans and policies of this kind is a genuine understanding of how improvements to the interconnectivity of the street network and the topological relationship between private and public space can generate vital urban activities in these areas.

In the Netherlands, there is a tendency to regenerate whole residential areas thirty years after realisation. Often these areas are classified by the government as 'problem neighbourhoods' due to a high concentration of youngsters, immigrants, or the socially under-privileged. Most of these areas are post-war social housing areas with poorly integrated and unconstituted streets and with large numbers of semi-private spaces (Van Nes and López 2013). Resources are often put into restructuring these areas, but the focus is mostly on the intrinsic properties of space in terms of changing the building materials and architectural styles, shining up the parks and the playgrounds between buildings, adding some new programmed activities into the neighbourhood, etc. (Van Nes and López 2013). Recently, a strategy has been to stimulate a gentrification process by enhancing a large variation of social composition of the residents. Houses are provided for residents from all social classes in society, from the rental apartments for low-income residents up to luxury apartments or houses for sale to high-income people. So far, these strategies have not proven to be successful. The key seems to be to deal with urban 'space' before implementing urban 'form' in these neighbourhoods.

The relevance of compactness for urban sustainability can be assessed more adequately in spatial configurative terms than in other less formal terms. This is because a space syntax approach has operational terms to distinguish the spatial parameters of the built environment as an object shaped by societal processes, and conversely how this object influences various socio-economic processes. An approach of this kind assesses in what way economic and social behaviours are influenced by spatial configurative changes, and conversely, how they, in turn, influence such changes. Urban compactness can be described in spatial configurative terms as a street and road network's various degrees of inter-accessibility and how this network is connected to the whole city on local and global scales.

A high density of streets within a short metric radius and their high degree of inter-accessibility on various scale levels in an urban street network contributes to vital urban centres and residential areas. It is not enough to just encourage high density in urban areas by increasing the number of dwellings and the locales for economic activities or just a generally higher density of the built mass. It is the density of the street network, its degree of interconnectivity between main routes and local street network, its degree of permeability and visibility from adjacent buildings, and its local and global position in the whole system that enhances walking and sustainable modes of mobility such as public transport. The density of lively residential areas seems to be a by-product of the density of the urban street network and the dispersal of integration values within the network.

If a compact urban area is conceived of as having a dense and well-connected street network, both on a local and on a global scale, and high intervisibility between buildings and streets, then compactness of this kind is a complex set of sufficient conditions for generating sustainable urban processes in terms of a low degree of energy use for transport (public transport, walkability). 
Compared with many other accounts of urban sustainability, a space syntax approach can offer specific concepts of spatial and functional aspects to explain or understand compact cities and their effects on economic and social behaviour-in other words, whether the built environment turns out to be sustainable or not. Generating sustainable mobility means from a spatial point of view a chain of coincidences, and there are several physical aspects of the built environment that have to be present at the same time.

High density of a highly connected and integrated street network within a short metric distance and high street intervisibility have to be present at the same time in order to generate walkability, street safety, and low energy use for transport (De Koning and Van Nes 2019a, b). If a street is highly integrated, but all buildings do not have entrances and windows on the ground floor towards the streets, this will negatively affect the degree of walkability and the perception of street safety. Blind walls and buildings turned away from streets are perceived to be unsafe.

Seemingly, the spatial structure of the street network on various scale levels matters for achieving some of the United Nations sustainable development goals. Reduced energy use for transport touches upon parts from goal 7 (affordable and green energy) and goal 11 (sustainable cities and communities). Walkability touches upon parts from goal 11 and 3 (good health and wellbeing). Streets enhancing gender balance touch upon goal 5 (gender equality), whereas safe streets and possibilities for micro-economic activities touch upon goal 11 .

\subsection{Exercises}

\section{Exercise 1}

Write down your research question(s). Reflect upon whether your research question has a descriptive or a normative approach.

\section{Exercise 2}

Given the following logical structure:

$p$ is a sufficient condition for $q$.

$p$ is a necessary condition for $q$.

Discuss which one of these two modal logics formulas is right for the following statements:

(a) High spatial integration of the street network is a condition for high pedestrian flow rates.

(b) The intention to reduce the clashes between Protestants and Catholics was a condition for the construction of the peace walls in Belfast.

(c) The removal of the Berlin Wall was a condition for the emergence of Berlin's main shopping street Friederichstraße.

(d) Placing an object in the middle of a square is a condition for segregating this square.

(e) A highly integrated street is a condition that shops will locate themselves along the street.

(f) Short urban blocks are a condition for generating more people in streets.

(g) Implementing long buildings is a condition for segregating urban space.

(h) The intention to preserve the old street structure is a condition for the conservation plan of the Friederichstad area in Berlin.

(i) A new road link is a condition for changes in the integration values of the street network.

(j) The intention to improve the accessibility to Trafalgar Square was a condition for implementing the new pedestrian plan.

(k) The intention to reach as many customers as possible is a condition that shops will locate themselves along the highest accessible streets in a city.

(1) The intention to relieve the town centre from through traffic was a Coventry.

(m) High density of the built mass is a condition for a high degree of land use diversity.

(n) The intention to implement the planning ideals from the CIAM 1933 conference was a condition for implementing a segregated street network in Oosterwei in Gouda.

(o) The Eiffel Tower is a condition for the city image of Paris. 


\section{Exercise 3}

Below are some complicated statements. Try to fill in whether it is a necessary or sufficient condition. Or where it is impossible to use either. Explain why.

(a) A landmark is a condition for a place's image.

(b) A compact city is a condition for generating sustainable mobility means.

(c) A dense urban street network is a condition for a high degree of walkability.

(d) A segregated street network was a condition for the high occurrence of crime in Oosterwei in Gouda.

(e) The lack of intervisibility of entrances and windows towards streets is a condition that women avoid frequenting these kinds of streets.

\section{Exercise 4}

Given the following advanced logical structures below:

Conjunction of a phenomenon: Maybe $p$ or $r$ alone is sufficient for $q$ to occur. However, if $p$ and $r$ occur together, $q$ is certain to occur too.

Disjunction of a phenomenon: Maybe $q$ does not require the presence of $P$ (unconditionally), nor the presence of $r$ (unconditionally); but $q$ may nevertheless require that at least one of the two, $p$ or $r$, be present.

Discuss below which of the above formulas is suitable for the statements below. Explain why you chose them. First, we show one example:

$p=$ straight lines, $r=$ partitioning a longer line, $q=$ increased depth.

Maybe increased depth does not require the presence of a partitioning of a longer line (unconditionally), nor the presence of straight lines (unconditionally); but increased depth may nevertheless require that at least one of the two, a partitioning of a longer line or straight lines, be present.

Here in this case the statement above is a disjunction of a phenomenon with a set of complex necessary conditions.

(a) $p=$ high spatial integration on all scale levels, $r=$ constituted streets, $q=$ Street life in London

(b) $p=$ high local integration, $r=$ high global integration, $q=$ high flow human movement through cities

(c) $p=$ high spatial integration, $r=$ high degree of function mixture, $q=$ high building densities

(d) $p=$ diverging political ideologies, $r=$ construction of the Berlin Wall, $q=$ disappearance of shops along the Friederichstraße in Berlin

(e) $p=$ spatial segregation, $r=$ low building densities, $q=$ mono-functional areas

(f) $p=$ the highest globally integrated road in Beijing, $r=$ Mao was an important leader for China for many years,

$q=$ location of the large picture of Mao at the main entrance of the Forbidden City in Beijing

(g) $p=$ street constitutedness, $r=$ high local and global integration, $q=$ high degree of walkability

(h) $p=$ road blockages, $r=$ new road link, $q=$ spatial configurative changes of the street network

\section{Exercise 5}

Describe what kind of approach your research belongs under. Is it positivistic or hermeneutics? Describe why?

\section{Exercise 6}

Describe what part of your city centre is a cultural centre or an economic centre.

\section{Exercise 7}

Describe the theoretical findings from your research. Do the results from your research explain a phenomenon or do they contribute to an understanding of a phenomenon? Give reasons as to why.

\section{Exercise 8}

Describe with your space syntax analyses what parts of your own city can be considered to be sustainable or not in terms of encouraging sustainable mobility means, safety, and street life. Identify at the end the parts where you have to use:

(a) necessary conditions

(b) sufficient conditions

(c) complex sufficient conditions (Conjunction of a phenomenon)

(d) complex necessary conditions (Disjunction of a phenomenon) 


\subsection{Answers}

\section{Exercise 2}

2a: sufficient, 2b: necessary, 2c: necessary, 2d: sufficient, 2e: sufficient, 2f: sufficient, $2 \mathrm{~g}$ : sufficient, $2 \mathrm{~h}$ : necessary, $2 \mathrm{i}$ : sufficient, $2 \mathrm{j}$ : necessary, $2 \mathrm{k}$ : sufficient, $2 \mathrm{l}$ : necessary, $2 \mathrm{~m}$ : sufficient, $2 \mathrm{n}$ : necessary, $2 \mathrm{o}$ : necessary.

\section{Exercise 3}

3a: neither, because it is not connected to a particular place.

$3 \mathrm{~b}$ : neither, because the term 'compact city' is vaguely defined.

$3 \mathrm{c}$ : neither, because walkability depends on the cultural background and other spatial or physical parameters such as pavements, street constitutedness, etc.

3d: neither, because there could be other spatial and social parameters.

3e: neither, because it is not connected to a particular place.

\section{Exercise 4}

Conjunction, 4b: Disjunction, 4c: Conjunction, 4d: Conjunction, 4c: Disjunction, 4f: Conjunction, 4g: Conjunction, 4h: Disjunction.

\section{References}

Aleksandrowicz, O, C. Yamu, and A. Van Nes. 2018. Spatio-syntactical analysis and historical spatial potentials: The case of Jaffa-Tel Aviv. The Journal of Interdisciplinary History XLIX (3): 1-28.

Alexander C. 1965. A city is not a tree. Architectural Forum $122(1+2): 58-62$.

Alexander, C., S. Ishikawa, M. Silverstein, M. Jacobson, I. Fiksdahl-King, and S. Angel. 1977. In A pattern language. New York: Oxford University Press.

Aleksandrowicz, O., C. Yamu, and A. van Nes. 2017. The socio-spatial development of Jaffa-Tel-Aviv: The emergence and fade-away of ethnic divisions and distinctions. In Proceedings of the 11th International Space Syntax Symposium Lisbon, ed. T. Heitor, M. Serra, J. P. Silva, M. Bacharel, and L. Cannas da Silva, (pp. 1-20). [140] Instituto Superior Técnico, Departamento de Engenharia Civil, Arquitetura e Georrecursos, Portugal.

Brundtland Commission. 1987. Our common future. Oxford: Oxford University Press.

Bruyns, G., A. Van Nes, C. Pinilla, R. Rocco, and J. Rosemann. 2007. The fifth city. In International Forum Urbanism, TU-Delft, Delft.

Calthorpe, P. 1993. The next American metropolis. Ecology, community, and the American dream. New York: Princeton Architectural Press.

Cuthbert, A.R. 2007. Urban design: Requiem for an era-Review and critique of the last 50 years. Urban Design International 12: $177-223$.

De Koning, R.E., A. Van Nes, Y. Ye, and H.J. Roald. 2017. Strategies for integrated densification with urban qualities. Combining Space Syntax with building density, land usage, public transport and property rights in Bergen city. In Proceedings of the 11th international space syntax symposium, ed. T. Heitor, M. Serra, J.P. Silva, M.B. Carreira, L.C. Da Silva, and E. Bazaraite, University of Lisbon, Lisbon.

De Koning, R.E., and A. Van Nes. 2019. Urban space and energy usage. In Proceedings of the 12th international space syntax symposium, ed. S. Qiang et al., Beijing Jiao Tong University, Beijing.

De Koning, R.E., and A. Van Nes. 2019. How two divergent ideologies impact the location of functions in relation to spatial integration in arctic settlements. In Proceedings of the 12th international space syntax symposium, ed. S. Qiang et al., Beijing Jiao Tong University, Beijing.

Desyllas, J. 2000. The relationship between urban street configuration and office rent patterns in Berlin. PhD thesis, The Bartlett, University College London.

Encyclopaedia Britannica. 1955. In Encyclopaedia Britannica: a new survey of universal knowledge, vol. 21, Sorde - Texti. Chicago: W. Benton Publishers.

Falleth, E., K. Kollbotn, and E. Tombre. 1995. Land use development along by-pass roads. Technical report, NIBR.

Føllesdal, D., L. Walløe, and J. Elster. Argumentasjonsteori, språk og vitenskapsfilosofi. Universitetsforlaget, Oslo, 6th ed.

Gehl, J. 2010. Cities for people. Washington, Covelo, London: Island Press.

Hardner, H., A. Van Nes, A. Sorgenfri Jensen, K. Hegnre Reinau, and M. Weber. 2012. Time use and movement behaviour of young people in cities. The application of GPS tracking in tracing movement pattern of young people for a week in Aalborg. In Proceedings of 8th international space syntax symposium, ed. M. Greene. Pontificia Universidad Católica de Chile, Santiago, Chile.

Hausleitner, B. 2010. Urban form and its impact on scope of action for people. Investigations on urban blocks in Paris. In 17th conference International Seminar on Urban Form, ISUF 2010, Hamburg.

Hillier, B. 1996. Space is the machine: A configurational theory of architecture. Cambridge, UK: Cambridge University Press.

Hillier, B. 1999a. Specifically architectural theory: A partial account of the ascent from building as cultural transmission to architecture as theoretical concretion. Bartlett School of Architecture, UCL.

Hillier, B. 1999b. Centrality as a process: Accounting for attraction inequalities in deformed grids. Urban Design International 4 (3 \& 4$)$ : $107-$ 127.

Hillier, B. 2001. The theory of the city as object or how spatial laws mediate the social construction of urban space. In Proceedings of 3rd international space syntax symposium, ed. J. Peponis, J. Wineman, and S. Bafna, Atlanta. 
Hillier, B., and J. Hanson. 1984. The social logic of space. Cambridge, UK: Cambridge University Press.

Hillier, B., A. Penn, J. Hanson, et al. 1993. Natural movement: Or, configuration and attraction in urban pedestrian movement. Environment and Planning B: Planning and Design 20: 29-66.

Hillier, B., A. Penn, D. Banister, and J. Xu. 1998. Configurational modelling of urban movement network. Environment and Planning B: Planning and Design 25: 59-84.

Hillier, B., and C.F. Shu. 2000. Crime and urban layout: The need for evidence. In Secure foundations. Key issues in crime prevention, crime reduction and community safety, ed. S. Ballintyne, K. Pease, and V. McLaren. Institute for Public Policy Research, London.

Hillier, B., and Ö. Sahbaz. 2005. High resolution analysis of crime patterns in urban street networks: An initial statistical sketch from an ongoing study of a London borough. In Proceedings of 5th international space syntax symposium, ed. A. Van Nes, Delft.

Hillier, B. 2012. The genetic code for cities: Is it simpler than we thought? In Complexity theories of cities have come to age: An overview with implications to urban planning and design, ed. J. Portugali, H. Meyer, E.H. Stolk, and E. Tan. Heidelberg: Springer Complexity.

Hwang, I.S.Y. 2006. When does stacking become vertical sprawl? In The sustainable city IV. Urban regeneration and sustainability, ed. U. Mander, C.A. Brebbia, and E. Tiezzi, Wessex.

Jacobs, J. 1960. The death and life of great American cities. New York: Random House.

Jenks, M., E. Burton, and K. Williams. 1996. In The compact city. A sustainable urban form? New York: E \& FN Spon.

Lefebvre, H. 1991. The production of space, trans. D. Nicholson-Smith. Oxford: Blackwell Publishers Ltd.

Major, M.D, A. Penn, and B. Hillier. 1999. The urban village and the city of tomorrow revisited. In Proceedings of 2nd international space syntax symposium, ed. F. Holanda, Brasil.

Miranda JV, and A. van Nes. 2020. Sexual violence in the city: space, gender, and the occurrence of sexual violence in Rotterdam. Sustainability. 12(18): 7609. https://doi.org/10.3390/su12187609

Mohamed, A., A. Van Nes, and M.A. Salheen. 2015. Beyond informality: Traders as space experts in their own informal settlements. In Proceedings of the 10th international space syntax symposium, ed. K. Karimi, L. Vaughan, K Sailer, G. Palaiologou, and T. Bolton, 135:1135:19, Space Syntax Laboratory, The Bartlett School of Architecture, UCL, London

Moudon, A.V. 1997. Urban morphology as an emerging interdisciplinary field. Urban Morphology 1: 3-10.

Newman, O. 1972. In Defensible space; Crime prevention through urban design. New York: Macmillan.

Nguyen, T.M., and A. Van Nes. 2014. Identifying the spatial parameters for differences in gender behaviour in built environments. The flaneur and flaneuwe of the 21st century. In Che "genere" di città per il futuro, vol. 6 n. 10 (Giugo 2013), Universita degli studi di Napoli Federico II, Centro interdipartimentale L.U.P.T.

Norberg-Schulz, C. 1967. Intensjoner i arkitekturen. Universitetsforlaget, Oslo.

Norberg-Schulz, C. 1971. Mellom jord og himmel. En bok om steder og hus. Universitetsforlaget, Oslo

Norberg-Schulz, C. 1980. Genius Loci. New York: Rizzoli International Publications INC.

Næss, P. 2006a. Unsustainable growth, unsustainable capitalism. Journal of Critical Realism 5: 197-227.

Næss, P. 2006b. Urban structure matters. London: Routledge.

Popper, K. 1963. In Conjectures and refutations: The growth of scientific knowledge. Routledge.

Rocco, R., and A. Van Nes. 2005. The location of advanced producer services and urban change: A space syntax approach. In Proceedings of 5th international space syntax symposium, ed. A. Van Nes, Delft.

Roger, R. 1999. Towards an urban renaissance. New York: E \& FN Spon.

Rossi, A. 1983. The architecture of the city. Cambridge, Massachusetts: MIT Press.

Rueb, L., and A. Van Nes. 2009. Spatial behaviour in Dutch dwelling areas. How housing layouts affects its users' behaviour. In Proceedings 7th international space syntax symposium, ed. D. Koch, L. Markus, and J. Steen, Stockholm.

Rønneberg Nordhov, N.A., P. Weebe Ritland, A. Larsen, and A. Van Nes. 2019. The role of building entrances towards streets and the perception of safety in six neighbourhoods in Bergen. In Proceedings of the 12th international space syntax symposium, ed. S. Qiang et al., Beijing Jiao Tong University, Beijing.

Rådberg, J. 1996. Towards a theory of sustainability and urban quality: A new method for typological urban classification. In 14th conference of the international association for people-environment studies, ed. M. Gray, 384-392, Stockholm.

Saglie, I.L. 1998. Density and town planning: Implementing a densification policy. PhD thesis, Oslo School of Architecture.

Seamon, D. 1994. The life of the place. Nordisk Arkitekturforskning 1: 35-48.

Seamon, D. 2011. Place, place identity, and phenomenology: A triadic interpretation based on J. G. Bennet's systematics. In The role of place identity in the perception, understanding, and design of the built environment, ed. H. Casakin, 1-16. Betham Science Publishers.

Seamon, D. 2012. Merleau-Ponty, perception, and environmental embodiment: Implications for architectural and environmental studies. In Carnal echoes: Merleau-Ponth and the flesh of architecture, ed. R. McCann and P.M. Locke, 1-16. Routledge.

Seamon, D. 2020. In Memoriam: Bill Hiller (1937-2019). Environmental and Architectural Phenomenology 31 (1).

Vaughan, L., and A. Penn. 2001. The jewish 'ghetto' - formations and spatial structure. In Proceedings of 3rd international space syntax symposium, ed. J. Peponis, J. Wineman, and S. Bafna, Atlanta.

Van Nes, A. 2002. Road building and urban change. The effect of ring roads on the dispersal of functions in Western European towns and cities. $\mathrm{PhD}$ thesis, Agricultural University of Norway.

Van Nes, A., and L. Aghabeik. 2015. Ethnic groups and spatial behaviour in Rotterdam's neighbourhoods. In K Karimi, L Vaughan, K Sailer, G Palaiologou \& T Bolton (Eds.), Proceedings of the 10th international space syntax symposium, 102:1-102:17. Space Syntax Laboratory, The Bartlett School of Architecture, UCL, London.

Van Nes, A., and L. de Rooij. 2015. The perceived safety and spatial behaviour in three different neighbourhoods in Rotterdam. In Proceedings of the 10th international space syntax symposium, ed. K. Karimi, L. Vaughan, K. Sailer, G. Palaiologou, and T. Bolton, 139:1-139:19, Space Syntax Laboratory, The Bartlett School of Architecture, UCL, London.

Van Nes, A. 2005. Typology of shopping areas in Amsterdam. In Proceedings 5th international space syntax symposium, ed. A. Van Nes, Delft. 
Van Nes, A. 2017. What is the explanatory power of space syntax theory? The application of modal logics from theory of science. In Proceedings of the 11th international space syntax symposium, ed. T. Heitor, M. Serra, J.P. Silva, M.B. Carreira, L.C. Da Silva, and E. Bazaraite, University of Lisbon, Lisbon.

Van Nes, A., M. Berghauser Pont, and B. Mashhoodi. 2012. Combination of Space syntax with spacematrix and the mixed use index: The Rotterdam South test case. In Proceedings of the 8th international space syntax symposium, PUC, Santiago de Chile.

Van Nes, A., and M. López. 2010. Macro and micro scale spatial variables and the distribution of residential burglaries and theft from cars: An investigation of space and crime in the Dutch cities of Alkmaar and Gouda. Journal of Space Syntax (2).

Van Nes, A., and M. López. 2013. Spatial-socio classification of deprived neighbourhoods in the Netherlands. In Proceedings of 9th international space syntax symposium, ed. Y.O. Kim, H.T. Park, and K.W. Seo. Seoul, Korea: Sejong University Press.

van Nes A. 2021. The impact of the ring roads on the location pattern of shops in town and city centres. a space syntax approach. Sustainability, 13(7): 3927. https://doi.org/10.3390/su13073927

van Nes, A., and C. Yamu. 2018. Space Syntax: a method to measure urban space related to social, economic and cognitive factors. In The Virtual and the Real in Planning and Urban Design: Perspectives, Practices and Applications, ed. C. Yamu, A. Poplin, O. Devisch, and G. De Roo (pp. 136-150). (Routledge Research in Planning and Urban Design). Routledge, Taylor and Francis group.

von Wright, G.H. 1971. In Explanation and understanding. International Library of Philosophy and Scientific Method. London: Lowe \& Brydone Ltd.

Yamu, C. 2014. It is simply complex(ity). Modeling and simulation in the light of decision-making, emergent structures and a world of non-linearity. disP - The Planning Review 50(4): 43-53.

Yamu, C. 2020. Emerging and shifting centralities: Evidence from Vienna. In Raumplanung. Jahrbuch 2020: 50 Jahre Raumplanung an der TU Wien. Studieren - Lehren - Forschen, ed. T. Dillinger, M. Getzner, A. Kanonier, and S. Zech, Wien: Neuer Wissenschaftlicher Verlag (NWV), pp. 542-559.

Yamu, C., A. van Nes, and C. Garau. 2021. Bill Hillier's legacy: space syntax—a synopsis of basic concepts, measures, and empirical application. Sustainability, 13(6): 3394. https://doi.org/10.3390/su13063394

Ye, Y., and A. Van Nes. 2014. Quantitative tools in urban morphology: Combining space syntax, spacematrix and mixed-use index in a GIS framework. Urban Morphology 18 (2): 97-118.

\section{Further Readings}

Carmona, M., T. Heath, T. Oc, and S. Tiesdel. 2012. In Public places-Urban spaces. Routledge.

LeGates, R.T., and F. Stout. 2011. The city reader, 5th edn. Urban Reader series. Routledge.

Gehl, J. 1987. Life between buildings. London: Island Press.

Hillier, B., and J. Hanson. 1984. The social logic of space. Cambridge, UK: Cambridge University Press.

Hillier, B. 1996. Space is the machine: A configurational theory of architecture. Cambridge, UK: Cambridge University Press.

Jacobs, J. 1960. The death and life of great American cities. New York: Random House.

Marshall, S. 2005. Streets and patterns. Oxon: Spon Press.

Van Nes, A. 2012. Between heaven and earth. Christian Norberg-Schulz's contribution to the phenomenology of place and architecture. Environmental and Architectural Phenomenology 23 (1). Winter 2012.

von Wright, G.H. 1971. In Explanation and understanding. International Library of Philosophy and Scientific Method. London: Lowe \& Brydone Ltd.

van Nes, A., and C. Yamu. 2020. Exploring challenges in space syntax theory building: the use of positivist andhermeneutic explanatory models. Sustainability, 12(17): 7133. https://doi.org/10.3390/su12177133

Open Access This chapter is distributed under the terms of the Creative Commons Attribution 4.0 International License (http://creativecommons. org/licenses/by/4.0/), which permits use, duplication, adaptation, distribution and reproduction in any medium or format, as long as you give appropriate credit to the original author(s) and the source, a link is provided to the Creative Commons license and any changes made are indicated.

The images or other third party material in this chapter are included in the work's Creative Commons license, unless indicated otherwise in the credit line; if such material is not included in the work's Creative Commons license and the respective action is not permitted by statutory regulation, users will need to obtain permission from the license holder to duplicate, adapt or reproduce the material. 